\author{
Marquette University \\ e-Publications@Marquette
}

$1-28-2016$

\title{
Recent Advances in Development and Applications of the Mixed Quantum/Classical Theory for Inelastic Scattering
}

Dmitri Babikov

Marquette University, dmitri.babikov@marquette.edu

Alexander Semenov

Marquette University

Follow this and additional works at: https://epublications.marquette.edu/chem_fac

Part of the Chemistry Commons

\section{Recommended Citation}

Babikov, Dmitri and Semenov, Alexander, "Recent Advances in Development and Applications of the Mixed Quantum/Classical Theory for Inelastic Scattering" (2016). Chemistry Faculty Research and Publications. 516.

https://epublications.marquette.edu/chem_fac/516 


\title{
Recent Advances in Development and Applications of the Mixed Quantum/Classical Theory for Inelastic Scattering
}

\author{
Dmitri Babikov \\ Chemistry Department, Marquette University, \\ Milwaukee, WI \\ Alexander Semenov \\ Chemistry Department, Marquette University, \\ Milwaukee, WI
}




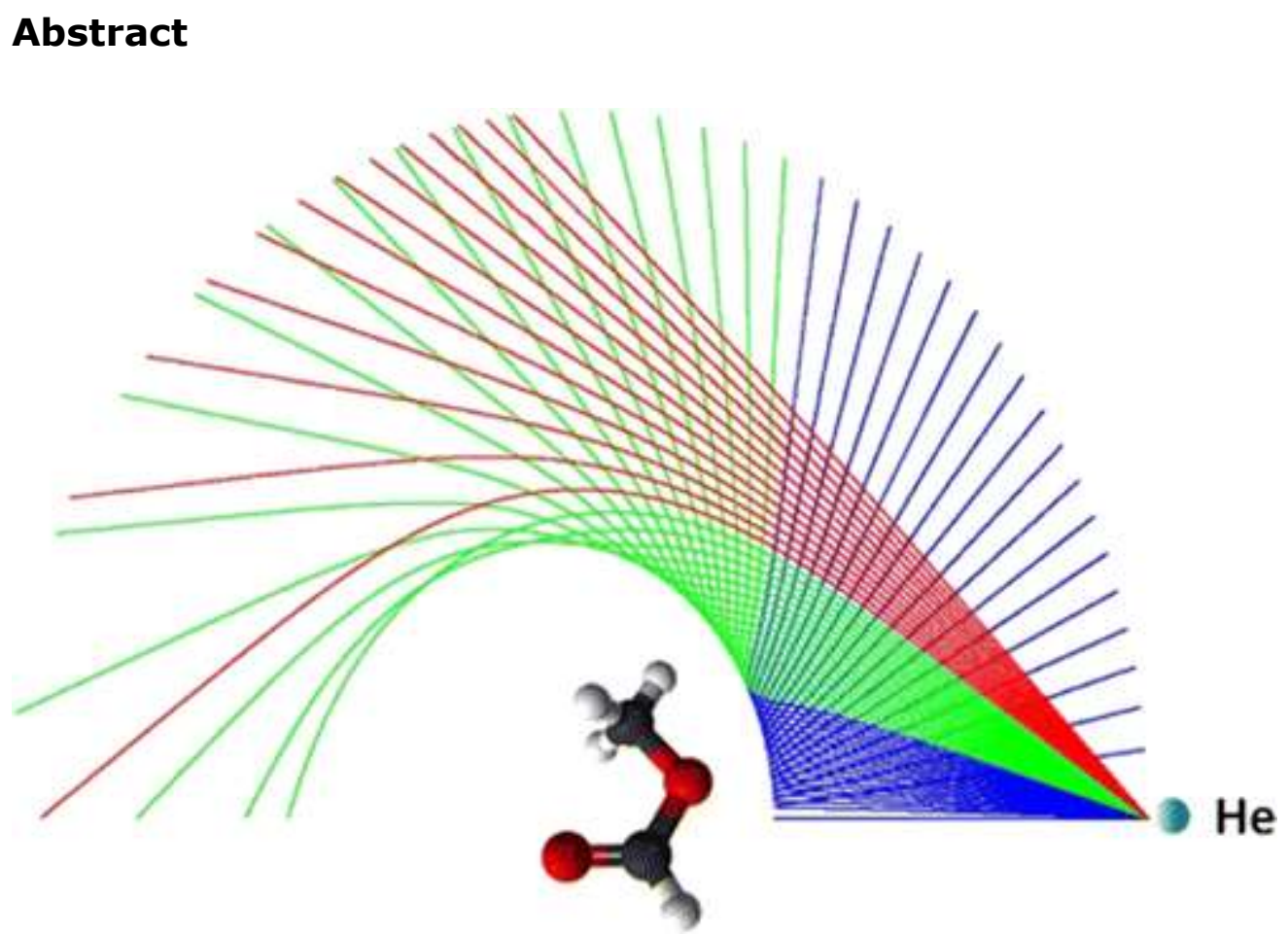

A mixed quantum/classical approach to inelastic scattering (MQCT) is developed in which the relative motion of two collision partners is treated classically, and the rotational and vibrational motion of each molecule is treated quantum mechanically. The cases of molecule + atom and molecule + molecule are considered including diatomics, symmetric-top rotors, and asymmetric-top rotor molecules. Phase information is taken into consideration, permitting calculations of elastic and inelastic, total and differential cross sections for excitation and quenching. The method is numerically efficient and intrinsically parallel. The scaling law of MQCT is favorable, which enables calculations at high collision energies and for complicated molecules. Benchmark studies are carried out for several quite different molecular systems $\left(\mathrm{N}_{2}+\mathrm{Na}, \mathrm{H}_{2}+\mathrm{He}, \mathrm{CO}+\mathrm{He}, \mathrm{CH}_{3}+\mathrm{He}, \mathrm{H}_{2} \mathrm{O}+\right.$ $\mathrm{He}, \mathrm{HCOOCH}_{3}+\mathrm{He}$, and $\mathrm{H}_{2}+\mathrm{N}_{2}$ ) in a broad range of collision energies, which demonstrates that MQCT is a viable approach to inelastic scattering. At higher collision energies it can confidently replace the computationally expensive full-quantum calculations. At low collision energies and for low-mass systems results of MQCT are less accurate but are still reasonable. A proposal is made for blending MQCT calculations at higher energies with full-quantum calculations at low energies.

\section{Introduction}

Collision of a molecule with another molecule (or an atom) in gas phase environment is a fundamental physical process important in 
a broad variety of chemical phenomena, ranging from astrochemistry that occurs on galactic sizes, ${ }^{1,2}$ to atmospheric chemistry, ${ }^{3,4}$ to combustion processes in the man-made engines, ${ }^{5,6}$ and to microfabricated traps cooled down to ultralow temperatures. ${ }^{7,8}$ At first glance the inelastic scattering process might look simple: two collision partners exchange energy by means of the interaction potential, but complexity is hidden in the details.

First of all, the energy is distributed between translational and internal degrees of freedom that include rotational and vibrational modes of one or both collision partners. Populations of these states may be far from thermodynamic equilibrium; so, detailed knowledge of many state-to-state transition cross sections is required. But in larger molecules the number of accessible internal states may reach hundreds and even thousands. ${ }^{9-12}$ Second, the molecule-molecule interaction is described by a complicated multidimensional potential, represented by the potential energy surface (PES), or hypersurface to be exact, that should be constructed using the tools of electronic structure theory and the state-of-the-art methods of surface fitting. ${ }^{13}$, ${ }^{14}$ Third, the range of scattering energies we have to cover is often broad, with different mechanisms dominating in different collision regimes (e.g., scattering resonances at low energies ${ }^{2,12,15}$ in contrast to vibrational excitation at high energies). ${ }^{16-18}$ Finally, very detailed information about the process may be needed, such as differential over scattering angle cross section for state-to-state transition. ${ }^{19,20}$ If several of these complications have to be tackled simultaneously, the inelastic scattering process does not look simple anymore.

One very popular theoretical tool for description of molecular collisions is the method of classical trajectories. ${ }^{21-23}$ It is relatively straightforward (in terms of computational methodology) and quite affordable (in terms of numerical cost) to deal with classical models of small polyatomic molecules as they rotate, vibrate, and exchange energy in a collision. We often use classical terms to visualize the collision process, and to understand it qualitatively. Problems with this approach occur at the final stage of calculations, when classical trajectories have to be analyzed to extract quantitative information, such as state-to-state cross sections. Experience shows that a classical description is appropriate only when the energy exchanged in the 
process is high, and the number of internal states involved (rotational, vibrational) is large. Examples include photochemistry, ${ }^{24}$ collisioninduced dissociation, ${ }^{25,26}$ exothermic chemical reactions, ${ }^{27}$ and hyperthermal phenomena. ${ }^{28}$ However, for the low-energy collisions, or when just a few low-lying quantum states are involved, the method of classical trajectories is not expected to be accurate. Moreover, several important molecular processes/features cannot be described by classical trajectories in principle, such as preservation of zero-point energy, ${ }^{29-31}$ symmetry restrictions for state-to-state transitions, ${ }^{32}$ tunneling, ${ }^{33}$ and scattering resonances. ${ }^{29}$

In contrast, the quantum mechanical approach to inelastic scattering, such as coupled-channel (CC) formalism, ${ }^{34,35}$ is the most universal, general and (besides numerical convergence issues) is considered to be exact, because the full-dimensional Schrodinger equation is numerically solved without any physical approximations. Unfortunately, the cost of such calculations grows dramatically for heavier collision partners, at higher collision energies, and for the molecules with dense spectra of the rotational and vibrational states. For example, the $\mathrm{CC}$ calculations of rotationally inelastic scattering for a triatomic + diatomic system, such as $\mathrm{H}_{2} \mathrm{O}+\mathrm{H}_{2}$ with collision energies up to $8000 \mathrm{~cm}^{-1},{ }^{36}$ are at the limit of the present day computing power. ${ }^{37}$ It appears that similar calculations for a triatomic + triatomic system, such as $\mathrm{H}_{2} \mathrm{O}+\mathrm{H}_{2} \mathrm{O},{ }^{38}$ or for a polyatomic + atom system, such as $\mathrm{CHOOCH}_{3}+\mathrm{He}^{12}$ are so computationally expensive that they are considered impractical for any useful range of collision energies (e.g., at room temperature). Furthermore, inclusion of the lowest vibrational modes, such as bending in $\mathrm{H}_{2} \mathrm{O}$, or torsion in $\mathrm{CHOOCH}_{3}$, would make these calculations even more expensive. A somewhat sad but honest conclusion is that a broad variety of molecular collision processes, which include chemically important triatomic and small polyatomic molecules, are well outside the reach of theorists today.

Expanding our predictive capabilities, even slightly, toward these more complicated systems would be quite beneficial. This is why the focus of our research is on development of an efficient theoretical method that would involve a physical approximation to ease calculations but would still remain reasonably detailed and accurate, at 
least in collision regimes where the full-quantum approach is not affordable anymore, whereas the purely classical approach is not accurate. Pictorially, our approach can be "positioned" between the purely classical and the full-quantum methods discussed above, because it combines both classical and quantum mechanics to treat different degrees of freedom in a molecular scattering problem. Namely, we still use quantum mechanics to describe the internal quantized states of molecules, but we also employ classical mechanics to describe their translational motion. This is an approximation, but we demonstrate below that this is a good one to make for almost any molecular system in almost any collision regime. If the limitations of this approach are understood and are easy to predict a priori, it may become a useful addition to the toolbox of theorists. Importantly, a very significant computational speed-up, compared to the fullquantum method, is achieved because the quantum treatment of continuum motion (scattering of heavy particles that typically requires a large number of partial waves) is avoided and is replaced by the mean-field trajectory. Still, the state-to-state transitions are described by the time-dependent Schrodinger equation, which incorporates quantization of states, symmetry, tunneling, and other attributes of quantum mechanics.

We call our approach the mixed quantum/classical theory (MQCT). It was not developed in isolation, because similar theories have been proposed in the past, and we built upon that previous work. Very relevant is the early work of McCann and Flannery ${ }^{39-41}$ in 1970 s and, of course, the intense work of Billing ${ }^{42,43}$ in the 1980 s and 1990s. The great work of Billing was so influential that at the end of $1990 \mathrm{~s}$ and beginning of 2000s quite a few theory groups around the world decided to give a try to the mixed quantum/classical approach, applying it to a broad variety of problems, including nonadiabatic phenomena, ${ }^{44}$ ro-vibrational excitation, ${ }^{45}$ femtosecond spectroscopy, ${ }^{46}$ collision-induced dissociation, ${ }^{47}$ photodissociation, ${ }^{48}$ and solvent effect. ${ }^{49}$ Some of the results obtained at that time were quite encouraging but because those systems, processes, and collision conditions were so diverse, it was hard to come out with general conclusions about accuracy, generality, and numerical efficiency of the method. To our best knowledge, no one else pursued a focused 
systematic study of the method, and after the death of Billing in 2003,50 these activities gradually declined.

Similarly to Billing, ${ }^{42,43}$ we differentiate between two distinct implementations of MQCT ideas. The first (more approximate) implementation restricts quantum mechanical treatment to the vibrational degrees of freedom only, describing rotational motion classically, same as translational motion. Potentially, this is a powerful approach, suitable for description of ro-vibrational processes with large vibrational and rotational excitations, including dissociation. The largest body of Billing's work was dedicated to development and testing of this approach. Recently, we applied similar methods to describe stabilization of scattering resonances at the final step of the ozone forming reaction, in $\mathrm{O}_{3} *+$ Ar collisions, looking at the isotope effects, ${ }^{51-53}$ but also in the benchmark study of ro-vibrational quenching in $\mathrm{CO}(\mathrm{v}=1)+\mathrm{He} .{ }^{54,55}$ However, our major focus has been on the second implementation of MQCT, in which all the internal degrees of freedom are treated quantum-mechanically (rotational and vibrational states on equal footing) and only the scattering is described classically. Clearly, this version is more rigorous and is more suitable for a systematic benchmark study. Surprisingly, it has never been properly tested. Billing himself applied it to only one system, $\mathrm{H}_{2}+$ $\mathrm{He},{ }^{43,56}$ focusing on transitions between the few lowest states at two values of collision energies. Those limited results were not particularly representative.

In our recent work, ${ }^{57-63}$ summarized in this Feature Article, we carried out a systematic development of theory for this second more rigorous version of $\mathrm{MQCT}$, incorporating phase information into the formalism (to compute elastic and differential over scattering angle cross sections), expanding MQCT onto the general case of an asymmetric-top rotor (suitable for description of polyatomic molecules), and including the case of molecule + molecule collisions (which makes this overall theory complete). Simultaneously, we conducted a rigorous benchmark tests of MQCT, applying it to six different molecular systems, computing elastic and inelastic, total and differential cross sections for rotational excitation and quenching, of light and heavy collision partners, with low and high levels of the initial excitation and in a broad range of collision energies from few 
wavenumbers up to $10^{4} \mathrm{~cm}^{-1}$. In one case we including vibrational states into the basis set expansion, which enables calculations of coupled ro-vibrational processes. We also studied one case when the rotational energy released by one collision partner is absorbed by rotation of the other partner, the so-called quasi-resonant energy transfer between two molecules. Among the molecules we studied there were such important as $\mathrm{H}_{2} \mathrm{O},{ }^{2,16,17,19,38}$ and such large as $\mathrm{HCOOCH}_{3}$ which, to our best knowledge, is the most complicated system ever considered for the inelastic scattering calculations. ${ }^{12}$ In all cases we compared our MQCT results against results of the fullquantum calculations carried out with MOLSCAT $^{64}$ or Hibridon. ${ }^{65}$

\section{Theory}

Here we review definitions and the final equations of motion for MQCT (without detailed derivations) and discuss how to set up the initial conditions for those and how to convert the results of calculations into observable quantities. This presentation summarizes derivations and generalizes results of several earlier theory papers on diatomic + atom, ${ }^{54,55,57,59,60}$ polyatomic + atom, $58,61,62$ and diatomic + diatomic ${ }^{63}$ systems. The point we convey here is that MQCT equations have the same form for any system of two collision partners, the difference is only in the meaning of indexes and in the structure of state-to-state transition matrix. Notations we use here are somewhat different from those used in our earlier papers but are chosen to emphasize the generality of MQCT and to make the equations most transparent and user-friendly.

\section{II.1 Quantum and Classical Degrees of Freedom}

Consider inelastic scattering of two collisions partners that can be either two molecules or a molecule and an atom. In either case, classical variables that describe the scattering process in MQCT are three coordinates $(R, \Theta, \Phi)$ of the vector $\mathbf{Q}$ that connects centers of mass of two collision partners. This is illustrated schematically in Figure 1 for the case of a diatomic + diatomic collision, but again, this description remains valid if one of the molecules is replaced by an atom, or if a collision of two polyatomic molecules is considered. For any system, the uppercase variables $R, \Theta$, and $\Phi$ give the position of 
the classical vector $\mathbf{Q}$ with respect to the laboratory-fixed reference frame, using the usual spherical polar system of coordinates.

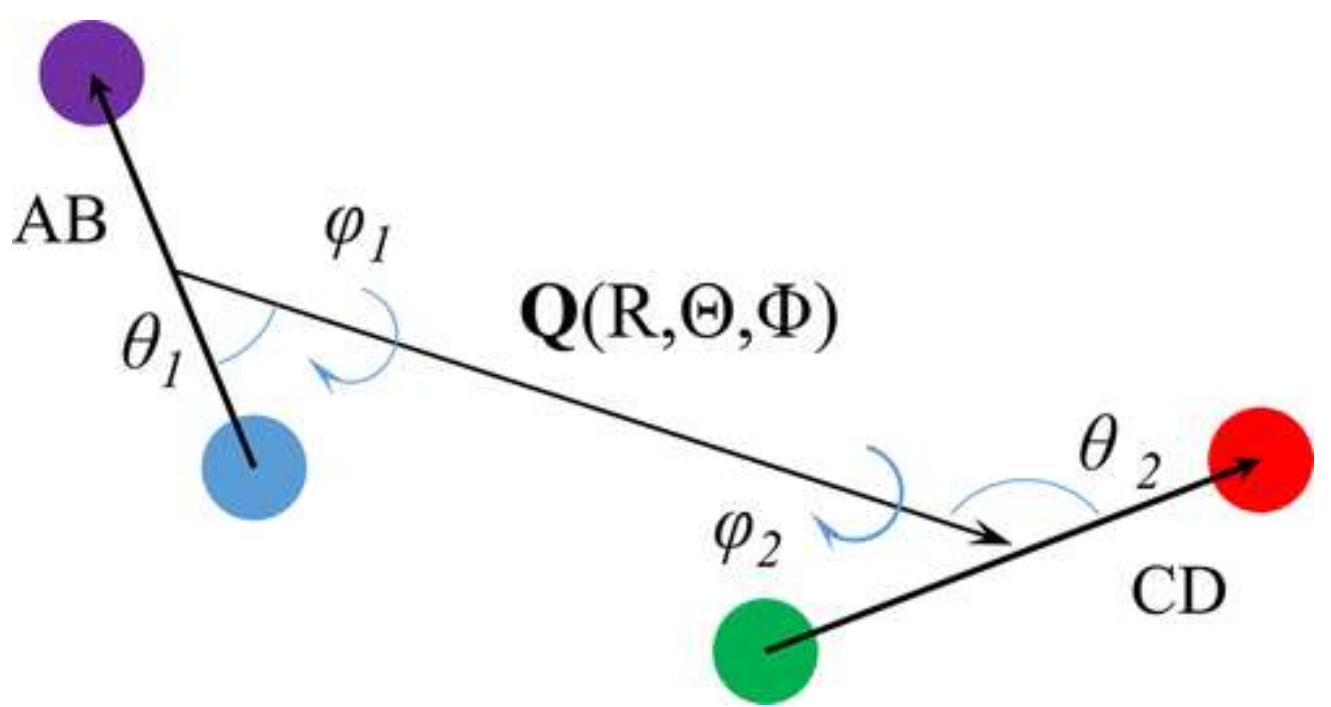

Figure 1. Classical and quantum degrees of freedom for description of inelastic collision of two diatomic molecules, $A B$ and $C D$, in the body-fixed reference frame.

Quantum degrees of freedom in MQCT are the angles needed to describe individual orientations of colliding molecules with respect to the vector $\mathbf{Q}$ (i.e., in the BF reference frame), and these are different for different systems. For example, in the diatomic + diatomic case depicted in Figure 1 these are four lowercase angles $\left(\theta_{1}, \theta_{2}, \varphi_{1}, \varphi_{2}\right)$. If the second molecule is replaced by an atom, then the second pair of angles is obsolete and only the first one is needed, just $(\theta, \varphi)$. If polyatomic molecule is considered, then we use Euler angles $(a, \beta, \gamma)$, for each molecule. For simplicity we will introduce a composite variable $\boldsymbol{\omega}$ to label all the quantum degrees of freedom in the system. At most, there are six angles needed for the general case of polyatomic + polyatomic collision, namely, $\boldsymbol{\omega}=\left\{\mathrm{a}_{1}, \beta_{1}, \mathrm{Y}_{1}, \mathrm{a}_{2}, \beta_{2}, \mathrm{Y}_{2}\right\}$.

The total time-dependent wave function for the quantum part of the system is expressed in MQCT as follows (in atomic units):

$$
\psi(\omega, t)=\sum_{m=1} a_{m,}(t) \Psi_{\min }(\omega) \mathrm{e}^{-\mathrm{i} E_{\mathrm{s}} t}
$$


where $a_{m n}$ are time-dependent expansion coefficients, $\Psi_{m n}$ is basis set of rotational eigenstates of the system, and $E_{n}$ are their corresponding energy eigenvalues. Index $n$ is a composite index that labels states and its meaning depends on the system. For the simplest case of a diatomic + atom we have simply $n \equiv\{j\}$ and $\Psi_{m n}(\boldsymbol{\omega}) \equiv Y_{j}^{m}(\theta, \varphi)$, i.e., spherical harmonics. ${ }^{57}$ For an asymmetric-top rotor (general polyatomic molecule) + atom we should set $n \equiv\left\{j, k_{a}, k_{c}\right\}$ and determine wave functions $\Psi_{m n}(\boldsymbol{\omega}) \equiv \Psi_{m j k a k c}(a, \beta, \gamma)$ by diagonalization of the rotational Hamiltonian in the basis set of Wigner $D$-functions. ${ }^{61}$ In either case, the energy $E_{n}$ of eigenstate depends on $n$ only and does not depend on $m$, which is a projection of angular momentum vector $\mathbf{j}$ of the molecule onto vector $\mathbf{Q}$, which plays the role of $z$-axis in the BF reference frame. For a diatomic + diatomic case depicted in Figure 1 we should set $n \equiv\left\{j_{1} j_{1}, j_{2}\right\}$, where $j_{1}$ and $j_{2}$ are individual angular momenta of the molecules, but now $j$ represents the total angular momentum of two molecules, $\mathbf{j}=\mathbf{j}_{1}+\mathbf{j}_{2}$, which is also quantized in MQCT: $\left|j_{1}-j_{2}\right| \leq j \leq j_{1}+j_{2}$. Corresponding eigenstates $\Psi_{m n}(\boldsymbol{\omega}) \equiv$ $\Psi_{m j j 1 j 2}\left(\theta_{1}, \theta_{2}, \varphi_{1}, \varphi_{2}\right)$ are expanded over basis set of spherical harmonics of two molecules using Glebsch-Gordan coefficients. ${ }^{63}$ The meaning of $m$ is still a projection of $\mathbf{j}$ onto $\mathbf{Q}$.

\section{2 Equations of Motion}

Substitution of eq 1 into time-dependent Schrödinger equation leads to MQCT equations for time-evolution of the quantum probability amplitudes $a_{m n}(t)$ and for the classically treated degrees of freedom in the problem, $\{R(t), \Phi(t), \Theta(t)\}$. Here, we present just the final equations, adapted for the case when the initial rotational wave function $\psi(\boldsymbol{\omega}, t=0)$ is a rotational eigenfunction $\Psi_{m n}(\boldsymbol{\omega})$ rather than a general rotational wave packet. In such system the rotational wave function possesses cylindrical symmetry around the vector $\mathbf{Q}$, and the classical trajectory of motion $\{R(t), \Phi(t), \Theta(t)\}$ is restricted to one plane. We choose this plane to be the equatorial plane $\Theta=\pi / 2$, which greatly simplifies both classical and quantum equations of motion. In this case the time-dependent Schrodinger equation for atom-molecule scattering is reduced to the following system of coupled equations for probability amplitudes: 
NOT THE PUBLISHED VERSION; this is the author's final, peer-reviewed manuscript. The published version may be accessed by following the link in the citation at the bottom of the page.

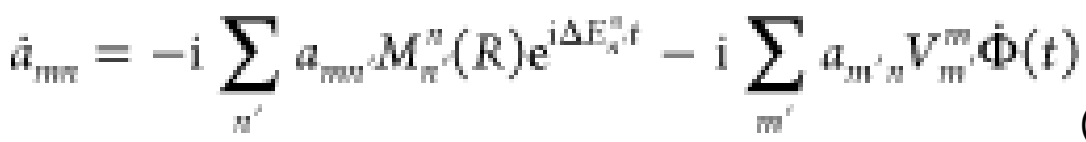

where $\Delta E_{n^{\prime}}{ }^{n}=E_{n}-E_{n^{\prime}}$. Here matrix $\mathbf{V}$ describes transitions between $m$ components of $j$ in the BF reference frame. It is computed analytically for every value of $j$ as follows:

$$
V_{m^{\prime}}^{m}=\frac{1}{2}\left[\sqrt{j(j+1)-m^{\prime}\left(m^{\prime}-1\right)} \delta_{m, m^{\prime}-1}+\sqrt{j(j+1)-m^{\prime}\left(m^{\prime}+1\right)} \delta_{m, m^{\prime}+1}\right]
$$

Note that matrix $\mathbf{V}$ is time-independent (should be computed only once) and is analytic. It does not involve any interaction potential. Physical meaning of the last term in eq 2 is the centrifugal coupling effect. Allowed transitions are $\Delta m= \pm 1$. The coupled-states (CS) approximation is obtained readily by neglecting this term. 57,60

In contrast, matrix $\mathbf{M}$ in eq 2 describes transitions between states $n$, and is computed separately for every $m$-component of $j$ using the potential energy surface $V(R, \boldsymbol{\omega})$ as follows:

$$
M_{m}^{H}(R)=\left\langle\Psi_{m i}(\omega)|V(R, o)| \Psi_{\text {inn }}(\omega)\right\rangle_{(4)}
$$

This is a potential coupling matrix and it should be computed numerically. Elements of $\mathbf{M}$ are real and depend on $R$ only, which is the length of the vector $\mathbf{Q}$, that itself evolves during the collision. For simple systems (diatomic, triatomic molecules) a useful expression for matrix elements can be obtained by expanding the interaction potential over a basis set of spherical harmonics. Such formulas for a diatomic molecule and symmetric-top and general asymmetric-top rotor have been published ${ }^{61}$ and will not be reviewed here. For more complicated (polyatomic) molecules it is better to compute matrix elements directly by multidimensional quadrature over $\boldsymbol{\omega}^{62}$ Equations of motion for classical degrees of freedom (coordinates of the vector $\mathbf{Q}$ ) are obtained using the Ehrenfest approach. ${ }^{57}$ The resultant differential equations for $R(t)$ and $\Phi(t)$ also include matrixes $\mathbf{M}$ and $\mathbf{V}$, as a commutator: $57,60,61$

$$
\dot{R}=\frac{P_{R}}{\mu}
$$

Journal of Physical Chemistry A, Vol 120, No. 3 (January 28, 2016): pg. 319-331. DOI. This article is (c) American Chemical Society and permission has been granted for this version to appear in e-Publications@Marquette. American Chemical Society does not grant permission for this article to be further copied/distributed or hosted elsewhere without the express permission from American Chemical Society. 


$$
\begin{gathered}
\dot{P}_{R}=-\sum_{m^{\prime} n^{\prime}} \sum_{m=n}\left(a_{m m^{\prime} n^{\prime}}\right)^{*} \cdot a_{m n} \mathrm{e}^{\mathrm{i} \Delta E E^{\prime} \mathrm{t}} \frac{\partial M_{n}^{n^{\prime}}(R)}{\partial R}+\frac{P_{\Phi}^{2}}{\mu R^{3}} \\
\dot{\Phi}=\frac{P_{\Phi}}{\mu R^{2}}(6 \mathrm{a}) \\
\dot{P}_{\Phi}=-\mathrm{i} \sum_{m^{\prime} n^{\prime}} \sum_{m n}\left(a_{m m^{\prime} n^{\prime}}\right)^{*} \cdot a_{m n} \mathrm{e}^{\mathrm{i} \Delta E_{n}^{n^{\prime} t}[\mathbf{M}, \mathbf{V}]_{m n}^{m^{\prime} n^{\prime}}}
\end{gathered}
$$

where $\mu$ is the reduced mass of two collision partners, and $\Delta E_{n}^{n^{\prime}}=E_{n^{\prime}}-$ $E_{n}$. We showed that expressions in right-hand sides of eqs $5 \mathrm{~b}$ and $6 \mathrm{~b}$ are real-valued, ${ }^{57}$ which leads to the real-valued momenta and their time derivatives. These classical-like equations can be propagated in time and space numerically, together with the quantum-like system of coupled eqs 2 for probability amplitudes, using a suitable numerical method, like fourth order Runge-Kutta.

\section{II.3 Initial Conditions and Final Analysis}

Setting up the initial conditions for MQCT calculations includes sampling over classical degrees of freedom, but calculations of the state-to-state transition cross sections incorporates sum over the final and an average over the initial degenerate states, just as in the fullquantum calculations. The goal is to keep MQCT as close as possible to the quantum formalism. For this reason we do not sample or use the classical collision impact parameter directly. Instead, we sample randomly and uniformly the value of $J$ that represents the total angular momentum in the problem, through the range $0 \leq J \leq J_{\max }$. The value of $J_{\max }$ is a convergence parameter, just as in the full-quantum calculations. For the randomly chosen value of $J$ and a chosen initial value of molecular angular momentum $j$ we sample the value of $l$ randomly and uniformly through the range $|J-j| \leq l \leq J+j$. It corresponds to the orbital angular momentum in the system, $\mathbf{I}=\mathbf{J}-\mathbf{j}$, and is used to define classical initial conditions as follows:

$$
\begin{gathered}
P_{\Phi}=\sqrt{I(l+1)}(7) \\
P_{R}=\sqrt{2 \mu E-P_{\Phi}{ }^{2} / R^{2}}(8)
\end{gathered}
$$

Journal of Physical Chemistry A, Vol 120, No. 3 (January 28, 2016): pg. 319-331. DOI. This article is @ American Chemical Society and permission has been granted for this version to appear in e-Publications@Marquette. American Chemical Society does not grant permission for this article to be further copied/distributed or hosted elsewhere without the express permission from American Chemical Society. 
where $E=k^{2} / 2 \mu$ is the kinetic energy of collision (not the total energy) and $R$ is the initial molecule-molecule separation (close to 20 Bohr). The initial value of $\Phi$ is arbitrary, and we use $\Phi=0$, as shown in the TOC graphic.

With these initial conditions MQCT equations are propagated through the collision event, until the point when the moleculemolecule separation exceeds the initial limiting value. The final values of probability amplitudes are used to compute transition probability (summed over the degenerate final states):

$$
\begin{aligned}
p_{\mathrm{m} n \rightarrow \mathrm{H}^{\prime}} & =\sum_{m^{\prime}\left(j^{\prime}\right)} p_{m u \rightarrow m^{\prime} n^{\prime}} \\
& =\sum_{m^{\prime}\left(j^{\prime}\right)}\left|a_{m^{\prime} n^{\prime}}\left(t_{\infty}\right)\right|^{2}
\end{aligned}
$$

Average of this quantity over the batch of $N$ sampled trajectories gives the corresponding cross section:

$$
\sigma_{m n \rightarrow N^{\prime}}=\frac{\pi}{k^{2}} \frac{J_{\max }}{N} \sum_{\text {trai }}(2 J+1) p_{m n \rightarrow n^{\prime}}
$$

The number of trajectories $N$ is also a convergence parameter. Note that sampling over $J$ and $I$ is done in a single step, because there is no requirement that the contribution of every $J$ is converged. It is only important that the entire sum of eq 10 is converged; so, we use a very efficient two-dimensional sampling that converges with a moderate number of MQCT trajectories (around 100 per initial state, per energy point). Finally, the cross section is averaged over the initial degenerate states, by running a set of $q$ independent calculations, taking each degenerate state as initial:

$$
\sigma_{n \rightarrow \pi^{\prime}}=\frac{1}{q} \sum_{m(j)} \sigma_{m n \rightarrow \pi^{\prime}}
$$

The entire procedure is, basically, the same for molecule + atom ${ }^{60-62}$ or molecule + molecule ${ }^{63}$ systems, except small differences in how the 
summation is done in eqs 9 and 11 . Namely, in the molecule + atom case, for each chosen initial $j$, the degenerate initial states are just the projection states $-j \leq m \leq+j$. So, in eq 11 the sum is over $m$ and the partition function is $q=2 j+1$. Similarly, in eq 9 the sum is over $m^{\prime}$, for each final $j^{\prime}$ of interest. However, in the molecule + molecule case, for a chosen pair of the initial states $j_{1}$ and $j_{2}$ (of two molecules) there are $q=\left(2 j_{1}+1\right)\left(2 j_{2}+1\right)$ degenerate states of the system. Those are labeled by $m$ varied in the range $-j \leq m \leq+j$ (for each allowed $j$ ) and by $j$, varied in the range $\left|j_{1}-j_{2}\right| \leq j \leq j_{1}+j_{2}$ So, in eq 11 the sum is over these $m$ and $j$, whereas in eq 9 the sum is over $m^{\prime}$ and $j^{\prime}$, allowed for each pair of the final states $j_{1}{ }^{\prime}$ and $j_{2}{ }^{\prime}$ of interest.

\section{4 Reversibility in MQCT}

Consider a transition $n \rightarrow n^{\prime}$ between two states of the system, characterized by a positive energy difference $\Delta E=\Delta E_{n}^{n^{\prime}}=E_{n^{\prime}}-E_{n}>0$, which corresponds to $E_{n^{\prime}}>E_{n}$. In general, calculations of cross section for this process can be set up by starting MQCT trajectories in state $n$ and looking at excitations into $n^{\prime}$ or, alternatively, by starting in state $n^{\prime}$, looking at quenching into $n$, and using the principle of microscopic reversibility. ${ }^{55}$ It states that if two such calculations are carried out at the same total energy $E_{\text {tot, }}$ the transition probabilities are equal:

$$
p_{n \rightarrow \pi^{n}}(E)=p_{n \rightarrow n^{3}}\left(E^{\prime}\right)
$$

In this expression $E$ and $E^{\prime}$ represent the initial kinetic energy of collision (Figure 2), related to the total energy as $E_{\text {tot }}=E_{n}+E=E_{n^{\prime}}+$ $E^{\prime}$. Unfortunately, this principle is not automatically built into the MQCT formalism (similar to classical trajectory simulations, and in contrast to quantum mechanics, where it is satisfied rigorously). Our experience shows ${ }^{55}$ that straightforward MQCT calculations of excitation, $n \rightarrow n^{\prime}$, overestimate transition probability, whereas the calculations of quenching, $n^{\prime} \rightarrow n$, underestimate it. Indeed, in calculations of excitation and quenching for the same total energy we always have $E>E^{\prime}$ and $P>P^{\prime}$, where $P=(2 \mu E)^{1 / 2}$ and $P^{\prime}=\left(2 \mu E^{\prime}\right)^{1 / 2}$ are the initial values of classical momenta. Because, in general, the probability of state-to-state transition depends on momentum (the speed) of collision, the results will always be different in the 
calculations of excitation and quenching. The problem is more pronounced at lower collision energies and for transitions with larger quanta $\Delta E$. It is particularly severe near the threshold (for excitation), when $E \approx \Delta E$ and $E^{\prime} \ll \Delta E$, which gives $E \gg E^{\prime}$ and leads to very different collision speeds in two calculations, $P \gg P^{\prime}$, resulting in drastically different (sometimes by several orders of magnitude ${ }^{55}$ ) transition probabilities for excitation and quenching, $p_{n \rightarrow n^{\prime}}(E) \gg$ $p_{n^{\prime} \rightarrow n}\left(E^{\prime}\right)$

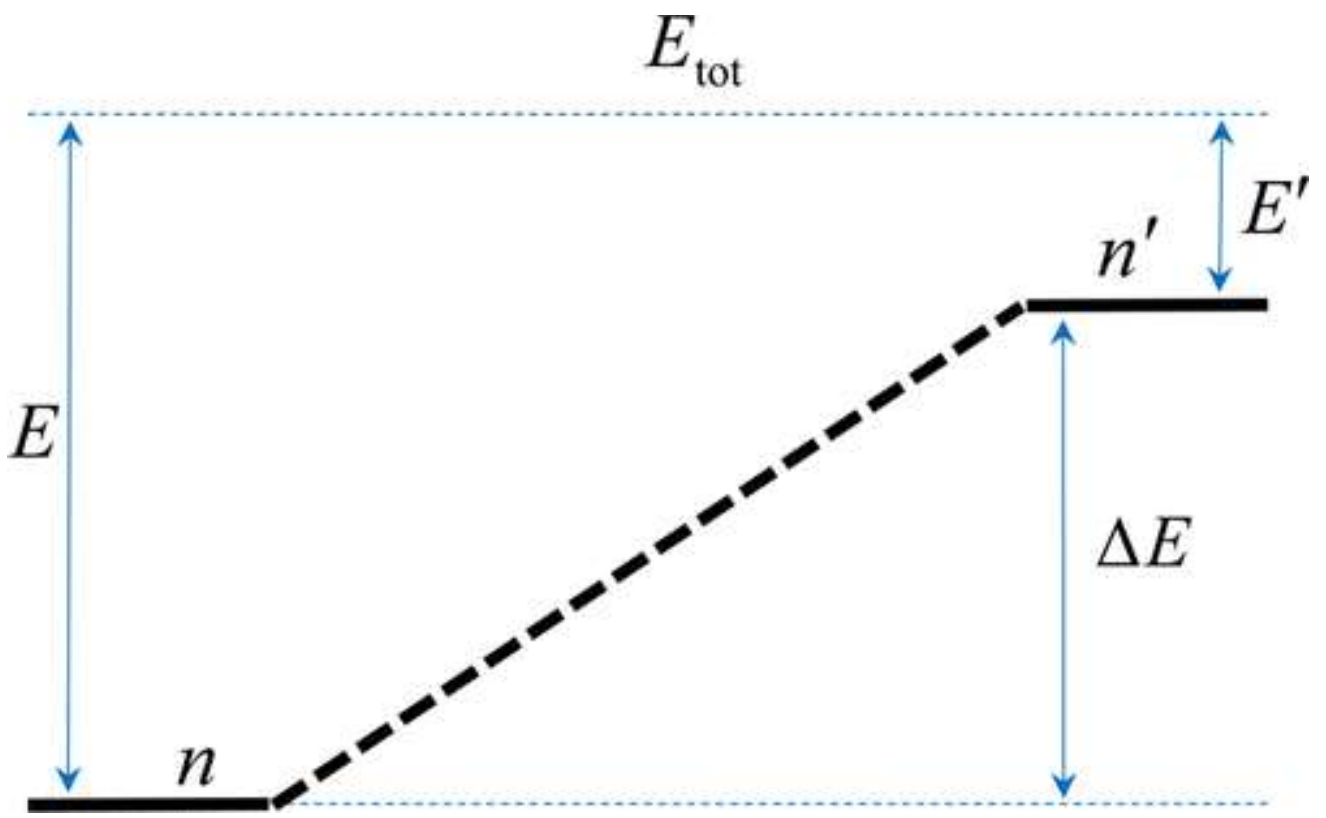

Figure 2. Schematic of energy balance in the principle of microscopic reversibility. $E$ and $E^{\prime}$ are kinetic collision energies in the calculations of excitation and quenching processes, respectively. See text for further details.

One proven way to enforce reversibility in MQCT is to run both excitation and quenching calculations with the same initial momentum that can be chosen as an average of $P$ and $P^{\prime} .{ }^{55,56}$ The corresponding effective collision energy $U$ is defined as

$$
\sqrt{U}=\frac{\sqrt{E}+\sqrt{E^{\prime}}}{2}
$$

It does not appear in any equations listed above but is used to set up the initial conditions in the actual calculations (namely, in eq 8 instead of $E$ ). Using simple algebra, one can express $U, E$, and $E^{\prime}$ through each other: ${ }^{55,56}$

Journal of Physical Chemistry A, Vol 120, No. 3 (January 28, 2016): pg. 319-331. DOI. This article is @ American Chemical Society and permission has been granted for this version to appear in e-Publications@Marquette. American Chemical Society does not grant permission for this article to be further copied/distributed or hosted elsewhere without the express permission from American Chemical Society. 


$$
\begin{gathered}
E=U+\frac{\Delta E}{2}+\frac{\Delta E^{2}}{16 U}(14) \\
E^{\prime}=U-\frac{\Delta E}{2}+\frac{\Delta E^{2}}{16 U}(15) \\
U=\frac{E+\sqrt{E(E-\Delta E)}}{2}-\frac{\Delta E}{4}(16) \\
U=\frac{E^{\prime}+\sqrt{E^{\prime}\left(E^{\prime}+\Delta E\right)}}{2}+\frac{\Delta E}{4}
\end{gathered}
$$

Note that $E^{\prime}<U<E$. Also note that at the threshold for excitation, when $E=\Delta E$ and $E^{\prime}=0$, we obtain $U=\Delta E / 4$. This means that the effective or actual collision energy $U$ of MQCT trajectories is never less than one-quarter of the quantum for the state-to-state transition of interest. This approach, equivalent to symmetrization of scattering matrix, allows merging the results of calculations for excitation and quenching, which enforces reversibility and significantly improves accuracy of MQCT, even in the regime of low collision energies and for the systems with large quanta $\Delta E .^{57}$ We followed this approach in all calculations of rotationally inelastic scattering presented in section III (six different molecular systems) and in our earlier calculations of rovibrational quenching in $\mathrm{CO}(v=1)+$ He system. ${ }^{57}$

\section{5 Scattering Phase and Differential Cross Section}

For the elastic scattering channel, and in particular for calculations of the differential (over scattering angle $\mathrm{X}$ ) cross section, the phase becomes important, but there are two contributions to the overall phase. The first contribution is phase acquired by the rotational wave function, the internal phase. It is contained in the complexvalued probability amplitude $a_{m n}$, which is accurately computed within MQCT. We denote this phase $\delta_{n}$ and can compute it simply as $\delta_{n}=$ $\arg a_{m n}\left(t_{\infty}\right)$. Second contribution is phase shift of the partial wave, ${ }_{i}$, which is missing in MQCT, because scattering is treated classically. However, classical treatment of translational degrees of freedom provides the deflection function $\chi^{(l)}$. It cannot really be used directly to

Journal of Physical Chemistry A, Vol 120, No. 3 (January 28, 2016): pg. 319-331. DOI. This article is @ American Chemical Society and permission has been granted for this version to appear in e-Publications@Marquette. American Chemical Society does not grant permission for this article to be further copied/distributed or hosted elsewhere without the express permission from American Chemical Society. 
compute the cross section at scattering angles smaller than the rainbow angle (see, for example, ref 66 ), but we found it possible to recover the value of $\delta_{i}$ from the $\chi(l)$ dependence. Namely, in the semiclassical treatments of scattering it is assumed that deflection is determined by the total phase shift:

$$
\chi(l)=\frac{\mathrm{d}}{\mathrm{d} l}\left(\delta_{l}(l)+\delta_{n}(l)\right)
$$

If the $\chi(l)$ and ${ }^{\delta_{n}}(l)$ dependencies are both known, this expression can be converted into differential equation for ${ }^{\delta_{i}(l)}$ with boundary condition $\delta_{l}(\infty)=0$, which corresponds to no scattering at large impact parameters. Solving such equation numerically allows reconstructing the ${ }^{\delta_{l}(l)}$ dependence:

$$
\delta_{l}(l)=-\delta_{n}(l)-\int_{\infty}^{l} \chi(l) \mathrm{d} l
$$

where $/$ is a dummy variable introduced for integration over $l$. This phase is then accounted for simply by multiplying the final computed probability amplitude by the phase factor: $a_{m u(}\left(t_{\infty}\right) \exp \left(i \delta_{i}\right)$. Such corrected probability amplitude can be used to compute the elastic scattering total cross section:

$$
\sigma_{m n \rightarrow m n}=\frac{\pi}{k^{2}} \frac{J_{\max }}{N} \sum_{\text {trai }}(2 J+1)\left|1-a_{m n n}\left(t_{\infty}\right) \exp \left(i \delta_{l}\right)\right|^{2}
$$

and the differential scattering amplitude (needed in calculations of the differential cross section $\left.{ }^{60}\right)$ :

$$
\begin{aligned}
f_{m u m}(\chi)= & \frac{\mathrm{i}}{k} \frac{J_{\max }}{N} \sum_{\text {traj }}(2 l+1)\left(1-a_{m u}\left(t_{\infty}\right) \exp \left(\mathrm{i} \delta_{l}\right)\right) \\
& P_{l}(\cos \chi)
\end{aligned}
$$

where ${ }^{P_{l}(\cos \chi)}$ is the Legendre polynomial of $l$ th degree. Note that this method can be applied to the inelastic scattering channels as well, but the total inelastic cross sections are insensitive to phases, because probability amplitudes are squared before any other operations in eq 
NOT THE PUBLISHED VERSION; this is the author's final, peer-reviewed manuscript. The published version may be accessed by following the link in the citation at the bottom of the page.

20 and the phase information is lost. For this reason we focused on the elastic channel.

\section{6 Few Remarks}

In the theory summarized above we neglected vibrational excitations and emphasized the (purely) rotational transitions, typical for molecules that are nearly rigid, described by the ground vibrational state wave function. But, if needed, the excited vibrational states can be easily added to the basis set expansion of eq 1 , by using rovibrational eigenstates, as we did in ref 60 . If the number of vibrational states is large, one can employ a grid-based approach, ${ }^{51}$ instead of the basis set expansion.

Another note concerns rotational variables. It is customary to use spherical polar angles $(\theta, \varphi)$ for diatomic molecules, whereas the Euler angles $(a, \beta, \gamma)$ are used for polyatomic molecules. However, for generality, the later could also be used instead of the former, because if we set $a=0$ then $\beta=\theta$ and $Y=\varphi$. We should also note that in several earlier papers we used primed variables and indexes for the BF reference frame (e.g., $\varphi^{\prime} m^{\prime}, a^{\prime}$ ) and unprimed for the SF reference frame. ${ }^{60,61,63}$ Such nomenclature is not followed here, simply because we did not discuss any BF-to-SF transformations in this paper, and to make the final MQCT equations most transparent. Also, using two angles $\varphi_{1}$ and $\varphi_{2}$ is redundant in some sense, because the potential of interaction of two molecules depends on their difference only, $\Delta \varphi=\varphi_{2}$ $-\varphi_{1}$.

\section{Numerical Results}

\section{1 Diatomic Molecules}

It was important to assess the accuracy of MQCT by comparing its results against the full-quantum results for simple systems. The first benchmark system we chose was $\mathrm{N}_{2}+\mathrm{Na}$, because it was studied recently in great detail by Dalgarno and co-workers ${ }^{20}$ using the fullquantum CC approach, and because the potential energy surface employed in that study was readily available. Figure 3 shows cross sections for rotational quenching and excitation of $\mathrm{N}_{2}(j=5)$ by 
collision with $\mathrm{Na}$ atom in a broad range of collision energies, $5<E<$ $1500 \mathrm{~cm}^{-1} .59,60$ We see that for the quenching processes, $5 \rightarrow 3$ and 5 $\rightarrow 1$, excellent agreement is observed between MQCT and the fullquantum calculations down to $E=5 \mathrm{~cm}^{-1}$, and even slight oscillations of cross sections (as a function of collision energy) are reproduced. For the excitation processes, $5 \rightarrow 7$ and $5 \rightarrow 9$, the agreement is also nearly perfect and even the channel thresholds are accurately reproduced (Figure 3). Here the MQCT method is accurate through 5 orders of magnitude range of cross section values. Similarly good agreement was also found for excitation of the ground rotational state $j=0$ of $\mathrm{N}_{2}$ into the states $j=2,4$, and $6 .{ }^{60}$

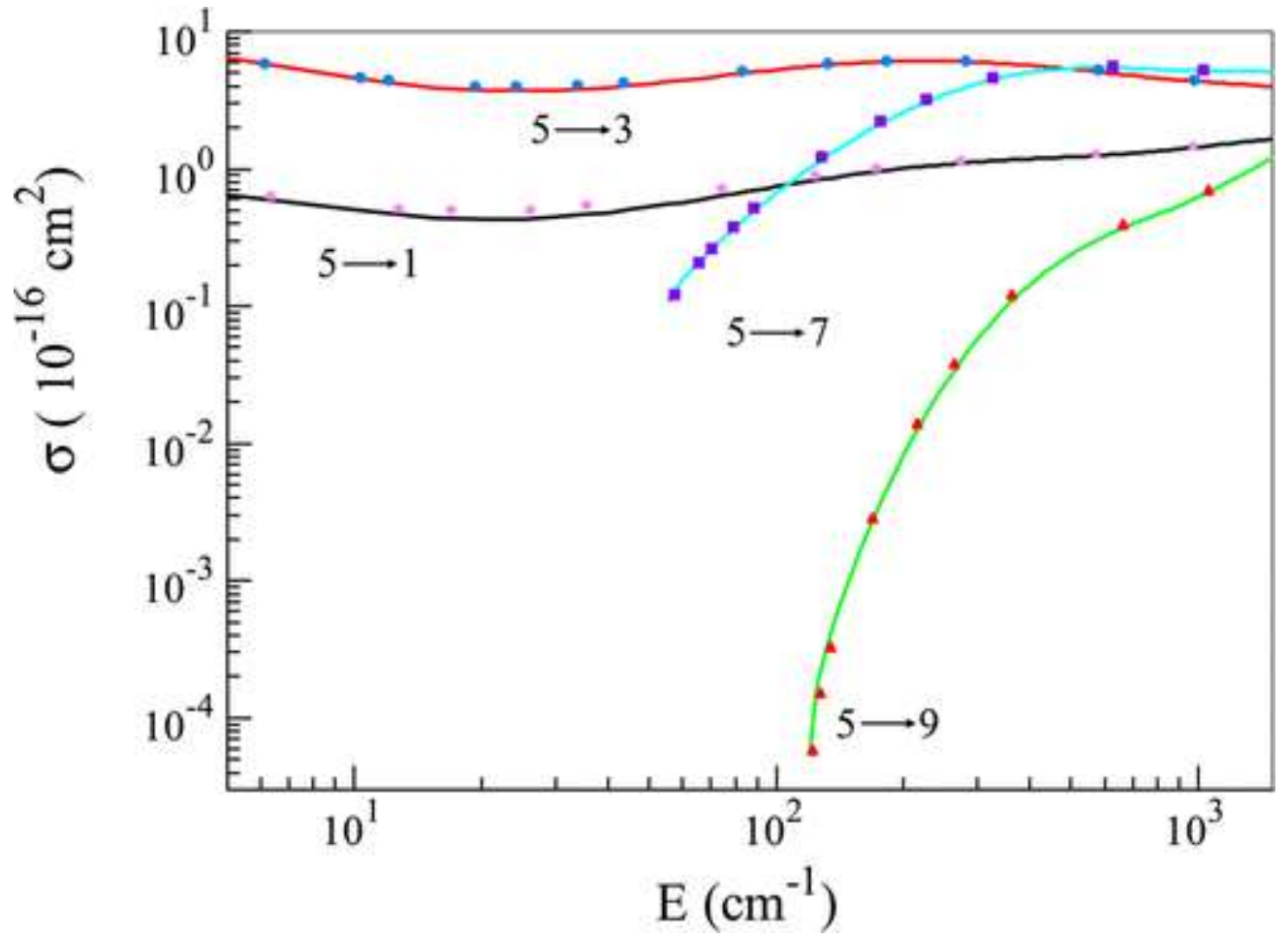

Figure 3. Inelastic scattering cross section for transitions in $\mathrm{N}_{2}+\mathrm{Na}$ from $j=5$ to $j=$ $1,3,7$, and 9. Full-quantum results of Dalgarno and co-workers ${ }^{20}$ are shown by lines; our MQCT results, ${ }^{59}$ by symbols.

Figure 4 shows differential cross sections for the elastic scattering channel $0 \rightarrow 0$ in the $\mathrm{N}_{2}+\mathrm{Na}$ system at collision energy $E=50 \mathrm{~cm}^{-1} .59$ Here, again, our MQCT results are tested against the full-quantum CC results of Dalgarno. ${ }^{20}$ The dependence is rather complicated, but every quantum oscillation is reproduced by MQCT, even at small scattering angles (forward scattering), where classical approximation is not 
expected to be accurate. Recall that neither the elastic nor the differential cross section can be reproduced by the classical scattering theory, due to the lack of phase information and quantum interference. ${ }^{66}$ But MQCT has it all and is accurate, again, through 5 orders of magnitude range of cross section values. Calculations of the differential cross sections at higher collision energies, 100 and $700 \mathrm{~cm}^{-}$ 1 , demonstrate excellent agreement too. ${ }^{60}$

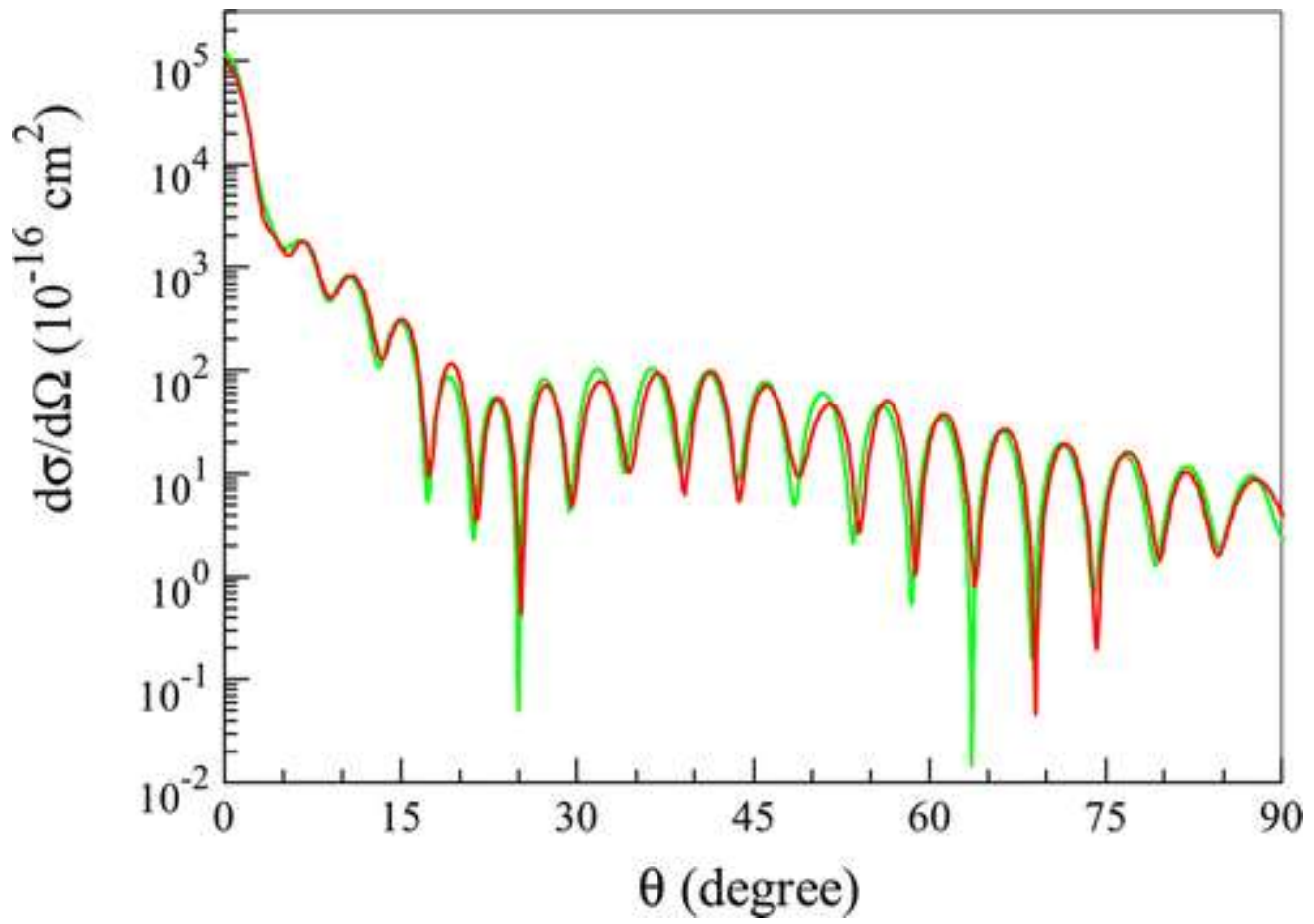

Figure 4. Differential cross section for elastic scattering of $\mathrm{N}_{2}(j=0)+\mathrm{Na}$ at $E=50 \mathrm{~cm}^{-1}$. Fullquantum results ${ }^{20}$ are shown by the green line; our MQCT results, ${ }^{59}$ by red line.

So, we see that for $\mathrm{N}_{2}+\mathrm{Na}$ collisions, MQCT is in detailed agreement with full-quantum calculations. It can be argued, however, that in this system all atoms are heavy, making it naturally suitable for classical treatment. This may be true, but one should keep in mind that all the chemically important atoms of the second and third rows of the periodic chart have masses comparable to those of $\mathrm{N}_{2}$ and $\mathrm{Na}$; so, this example is rather representative. Still, a test of MQCT using lighter atoms would be desirable to see how well the MQCT would work in such case.

For this reason we studied $\mathrm{H}_{2}+\mathrm{He}^{60}$ which is an all-light-atom system, often thought of as the most nonclassical example. In terms 
of the mass effect, it represents the most stringent test of MQCT.

Figure 5 gives the cross section for quenching of the first $(j=2)$ and second $(j=4)$ excited states of $\mathrm{H}_{2}$ by $\mathrm{He}$ impact through 4 orders of magnitude range of collision energies. Here comparison is against the full-quantum CC results of Balakrishnan, Stancil, and co-workers. ${ }^{8}$ In this system, indeed, we see some non-negligible differences between MQCT and the full-quantum calculations but, importantly, they vanish at collision energies above $100 \mathrm{~cm}^{-1}$, demonstrating accurate asymptotic behavior. Largest errors in MQCT calculations occur at lower energies. For example, at collision energies around $10 \mathrm{~cm}^{-1}$, predictions of MQCT for $\mathrm{H}_{2}+$ He exceed quantum results by $20 \%$ or so. For this system we also carried out calculations of quenching of the highly excited rotational state $\mathrm{H}_{2}(j=22)$ and we saw very similar behavior. ${ }^{60} \mathrm{~A}$ practically important conclusion is that even in the (worst) case of the lightest system, $\mathrm{H}_{2}+\mathrm{He}$, the MQCT method does not fail badly. Although less accurate, the results of MQCT remain reasonable, even at very low collision energies, on the order of just few wavenumbers (Figure 5). Needless to say that, for many applications, $20 \%$ error is acceptable. ${ }^{1}$

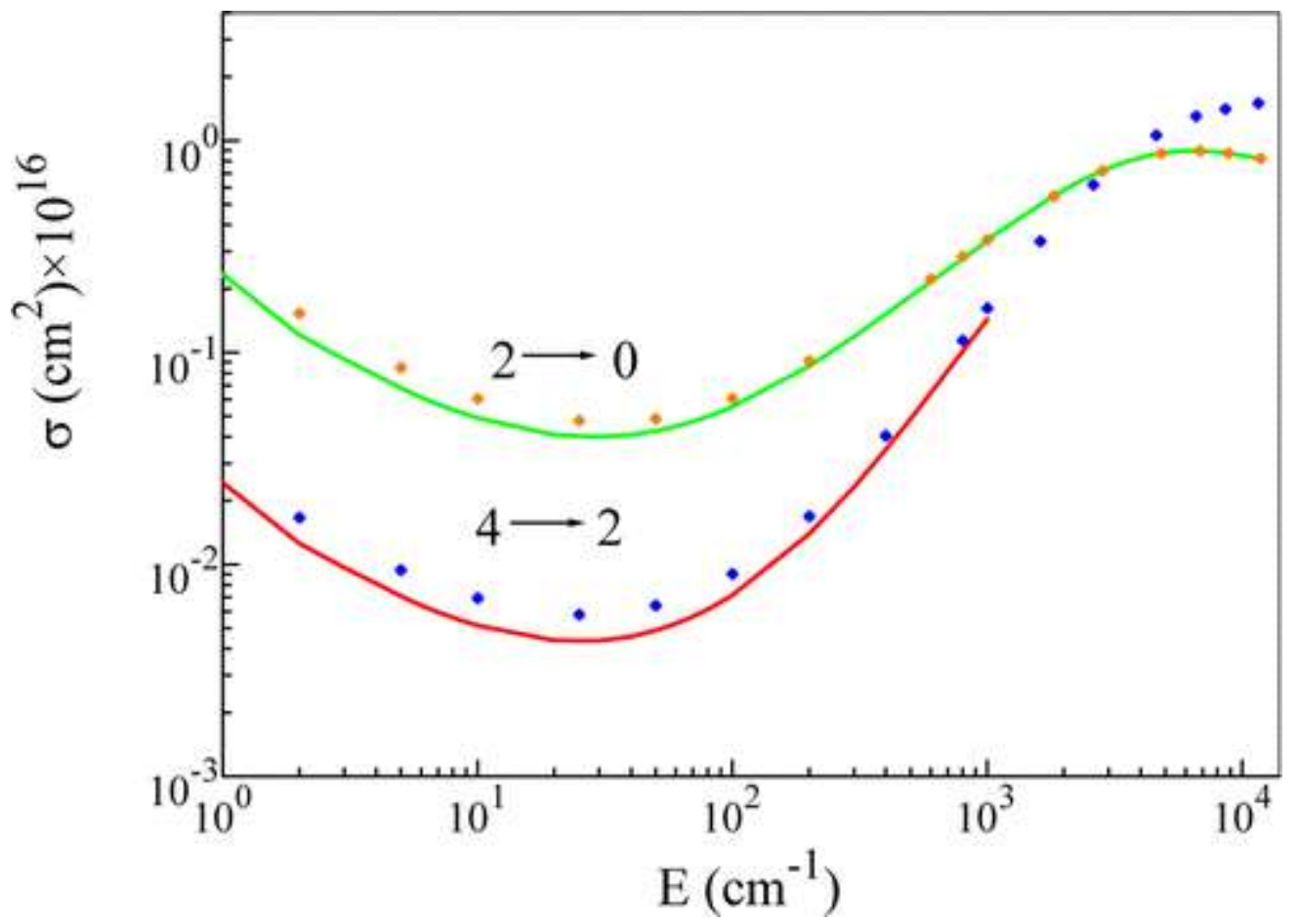

Figure 5. Quenching cross section for $\mathrm{H}_{2}+$ He. Full-quantum results of Balakrishnan, Stancil, and co-workers ${ }^{8}$ are shown by lines (where available); our MQCT results, ${ }^{60}$ )by symbols. Reprinted with permission from ref 60 (Semenov, A.; Dubernet, M.-L.;

Journal of Physical Chemistry A, Vol 120, No. 3 (January 28, 2016): pg. 319-331. DOI. This article is @ American Chemical Society and permission has been granted for this version to appear in e-Publications@Marquette. American Chemical Society does not grant permission for this article to be further copied/distributed or hosted elsewhere without the express permission from American Chemical Society. 
Babikov, D.Mixed Quantum/Classical Calculations of Total and Differential Elastic and Rotationally Inelastic Scattering Cross Sections for Light and Heavy Reduced Masses in a Broad Range of Collision Energies. J. Chem. Phys. 2014, 140, 044306). Copyright 2014 American Physical Society.

One more point we want to stress here is that MQCT permits rigorous incorporation of the symmetry selection rules for state-tostate transitions. Thus, in homonuclear diatomic molecules, such as $\mathrm{N}_{2}$ or $\mathrm{H}_{2}$, only the transitions with even values of $\Delta j$ are allowed. It is well-known that classical trajectory simulations produce a continuous spectrum of angular momentum values, and there is now a rigorous way of avoiding transitions that are quantum mechanically forbidden. In contrast, in MQCT the selection rules are enforced in the state-tostate transition matrix of eq 4 , by construction. Results presented above demonstrate this very clearly.

\section{2 Triatomic and Tetratomic Molecules}

The next natural step was to go beyond diatomic molecules by implementing MQCT for a symmetric-top rotor molecule collided with an atom. We chose a system of $\mathrm{He}+\mathrm{CH}_{3}$ (an oblate top), because it was studied recently by Alexander and co-workers ${ }^{5}$ using the fullquantum CC approach. Cross sections for rotational excitation of the ground state $\mathrm{CH}_{3}\left(j_{k}=0_{0}\right)$ into various excited states are presented in two frames of Figure 6, for collision energies up to $2500 \mathrm{~cm}^{-1}$. In this system we found that accuracy of MQCT may be different for different final states. For example, for the processes presented in the upper frame of Figure 6 the results of MQCT were in very good agreement with full-quantum results (similar to what we saw in diatomic molecules), which gave us confidence in the equations we derived and the code we wrote. But the lower frame of Figure 6 illustrates those transitions where the agreement was not that good. Although the general trend of energy dependence for each transition is captured by MQCT, we see that some difference between our MQCT results and the full-quantum results of Alexander ${ }^{5}$ survives even at higher energies (e.g., for transition $0_{0} \rightarrow 40$ it reaches $38 \%$ ). We did not see such behavior in others, in neither the simpler nor more complicated systems we studied so far, and do not quite understand the reason for this discrepancy yet (section III.4). 
NOT THE PUBLISHED VERSION; this is the author's final, peer-reviewed manuscript. The published version may be accessed by following the link in the citation at the bottom of the page.

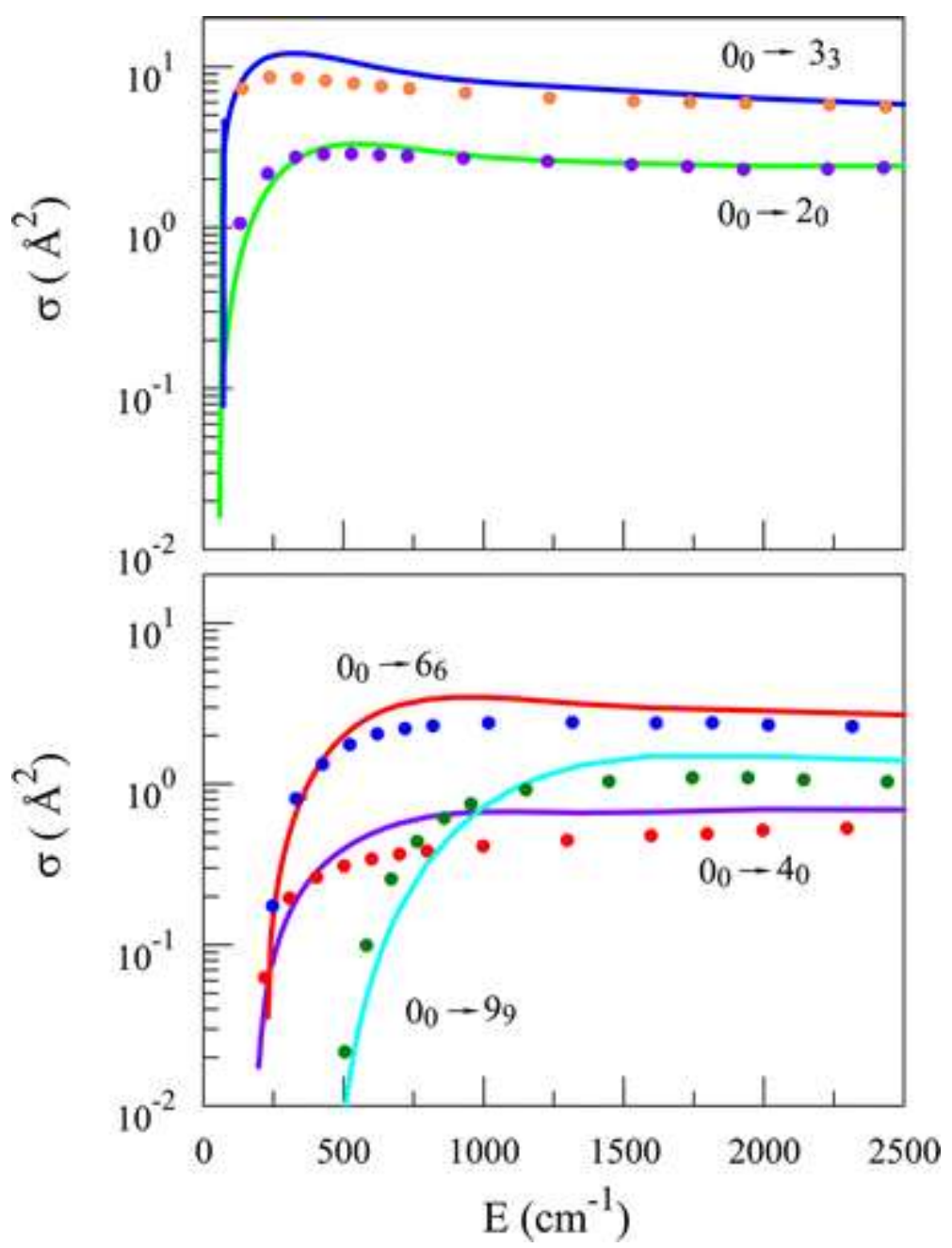

Figure 6. Energy dependence of rotational excitation cross sections for ground state of $\mathrm{CH}_{3}+\mathrm{He}$. Solid lines are full-quantum results of Alexander and co-workers; 5 symbols are our MQCT results.

To expand MQCT even further, onto the case of a general asymmetric-top rotor molecule, we carried out calculations for quenching of para- and ortho-water by He. ${ }^{58,61}$ Our results for quenching of several excited states $j_{k a k c}$ onto the ground state $0_{00}$ are shown in Figure 7. We see that, for water quenching, the results of MQCT are in very good agreement with full-quantum results (of Dubernet) in the entire range of considered energies, up to $10000 \mathrm{~cm}^{-}$ 1 , and through 4 orders of magnitude range of cross section values. At higher scattering energies results of MQCT coincide with full quantum results, for all transitions we studied. ${ }^{61}$ At lower energies the average error of MQCT is around 5-6\%. At scattering energies below $30 \mathrm{~cm}^{-1}$ errors on the order of $15 \%$ may occur. Interestingly, in the low-energy 
range, where broad scattering resonances are common, predictions of MQCT are still meaningful. They go through resonances in Figure 7 and represent the average (over resonances) value of the cross section. We also found that when resonances are narrow, numerous, and overlapping, the MQCT method describes well the nonresonant (background) behavior. Similar behavior was observed for $j$-changing and $k$-changing transitions between the rotationally excites states of water. ${ }^{61}$

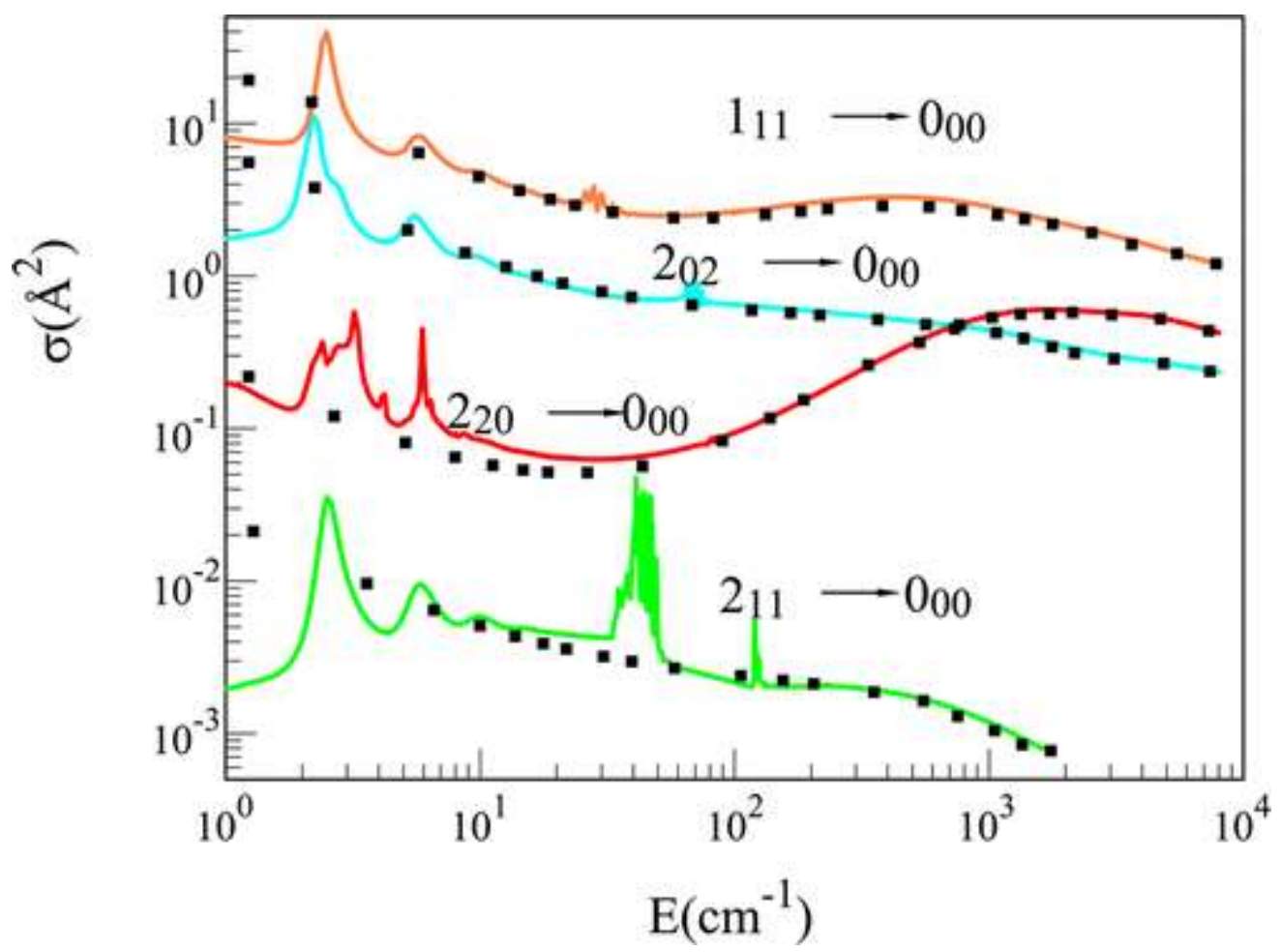

Figure 7. Rotational quenching cross sections of para- $\mathrm{H}_{2} \mathrm{O}$. Lines are full-quantum results of Dubernet; ${ }^{61}$ symbols are our MQCT results. Reprinted with permission from ref 61 (Semenov, A.; Dubernet, M.-L.; Babikov, D.Mixed Quantum/Classical Theory for Inelastic Scattering of Asymmetric-Top-Rotor + Atom in The Body-fixed Reference Frame and Application to the $\mathrm{H}_{2} \mathrm{O}+$ He System. J. Chem. Phys. 2014, 141, 114304). Copyright 2014 American Physical Society.

\section{3 Complex Systems}

Although $\mathrm{H}_{2} \mathrm{O}$ (discussed above) is a small triatomic molecule, it requires the most general treatment of molecular rotation, because it represents the case of an asymmetric-top rotor. Any other rigid polyatomic molecule, arbitrarily large, can be studied using the same theory and code. It is true that as polyatomic molecules become larger 
and larger, the assumption of rigidity holds less and less, due to possible excitations of floppy bending and large amplitude torsional modes. Still, purely rotational transitions in polyatomic molecules are of interest and are extremely hard to treat using the full-quantum approach, such as the CC method.

To our best knowledge, the largest molecule ever considered for such calculations is methyl formate, $\mathrm{HCOOCH}_{3}$, quenched by the He atom. Some full-quantum data for rotational excitation in this process are available from the work of Wiesenfeld and co-workers. ${ }^{12}$ Due to huge number of channels and partial waves (needed for convergence) the full-quantum CC calculations are affordable at very low collision energies only, below $30 \mathrm{~cm}^{-1}$. Another complication, quite typical for polyatomic molecules, is the difficulty of PES expansion over the basis set of spherical harmonics. It appears that, when the PES is complicated, too many expansion terms (hundreds) are required for accurate surface representation, and it is hard to truncate the expansion without introducing artifacts into the PES. ${ }^{9,12}$

In our MQCT calculations for $\mathrm{HCOOCH}_{3}+\mathrm{He}^{62}$ we used the same PES as in ref 12, but we did not expand it over the basis set of spherical harmonics. Instead, we computed matrix elements directly by integration of eq 4 over Euler angles. In Figure 8 we compared our MQCT results against the full-quantum results of Wiesenfeld ${ }^{12}$ for excitation of several lower states of methyl formate $j_{k a k c}$ starting from the ground state $0_{00}$, for the range of collision energies below $30 \mathrm{~cm}^{-1}$. We found that above $15 \mathrm{~cm}^{-1}$ the average difference of cross section values is about $5 \%$, which gives us argument to cautiously state that at higher energies MQCT is reasonably accurate for this system. In the lower energy range, $5-15 \mathrm{~cm}^{-1}$ in Figure 8 , results of MQCT for the most important transitions in $\mathrm{HCOOCH}_{3}$ are still reasonable. Only at collision energies below $5 \mathrm{~cm}^{-1}$, where quantum resonances dominate does the accuracy of MQCT drop significantly. At these low energies many MQCT trajectories describe orbiting of He atom around $\mathrm{HCOOCH}_{3}$, which is the classical analogue of scattering resonance. A good recipe for analysis of such trajectories is yet to be found. For now, we simply removed them from consideration, focusing on nonresonant contribution to the process. 
NOT THE PUBLISHED VERSION; this is the author's final, peer-reviewed manuscript. The published version may be accessed by following the link in the citation at the bottom of the page.

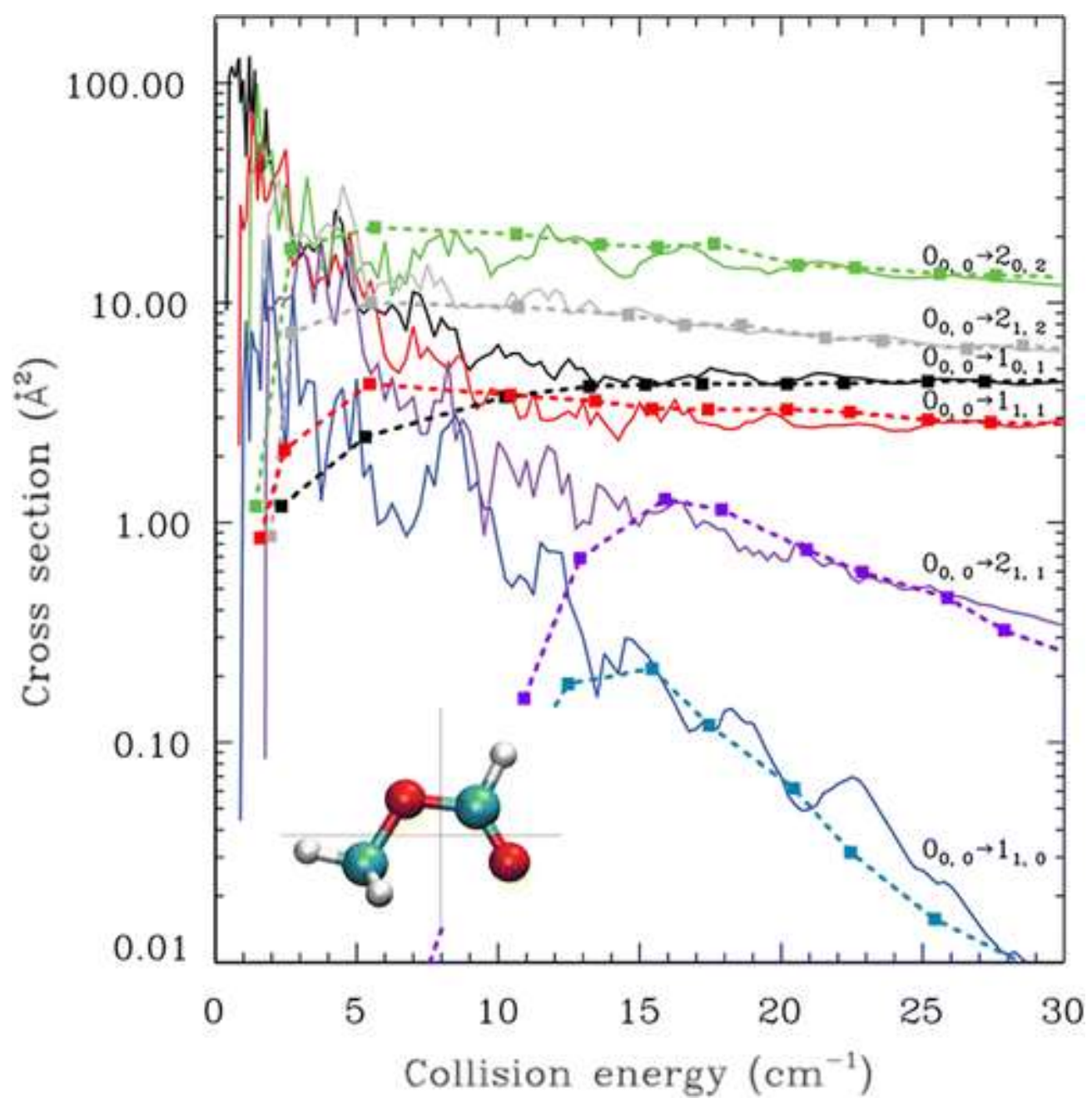

Figure 8. Cross sections for rotational excitation of methyl formate by collisions with the $\mathrm{He}$ atom, as a function of collision energy. Solid lines: full quantum results of Wiesenfeld and co-workers. ${ }^{12}$ Dashed lines/symbols: our MQCT results. ${ }^{62}$

Because MQCT is usually more accurate at higher energies, it makes sense to use it for prediction of excitation cross sections in $\mathrm{HCOOCH}_{3}$ at collision energies above $30 \mathrm{~cm}^{-1}$ (where there are no other data available). Thus, we computed cross sections for excitation of 20 most important rotational states of methyl formate at collision energies up to $1000 \mathrm{~cm}^{-1}, 62$ expanding the energy range by a factor of 33 compared to the previous work, ${ }^{12}$ which clearly demonstrates the efficiency of our approach.

So, it looks like rotational transitions in polyatomic molecules is one area where MQCT can be both efficient and accurate. Another important application would be in the molecule + molecule collisions.

Journal of Physical Chemistry A, Vol 120, No. 3 (January 28, 2016): pg. 319-331. DOI. This article is @ American Chemical Society and permission has been granted for this version to appear in e-Publications@Marquette. American Chemical Society does not grant permission for this article to be further copied/distributed or hosted elsewhere without the express permission from American Chemical Society. 
We started exploring this topic too, by looking at a diatomic + diatomic system (Figure 1 ). Most recently, ${ }^{63}$ we carried out MQCT calculations for rotational transitions in $\mathrm{N}_{2}+\mathrm{H}_{2}$, using the PES from ref 67. Because no one studied this system before, we also had to carry out the full-quantum CC calculations using MOLSCAT.

Comparison of our MQCT results against the full-quantum results for collision energies up to $4000 \mathrm{~cm}^{-1}$ is presented in Figure 9 for the process in which the rotationally excited $\mathrm{H}_{2}(j=2)$ excites the ground state $\mathrm{N}_{2}(j=0)$ into several upper states. We see that MQCT is accurate for $\mathrm{N}_{2}+\mathrm{H}_{2}$ in a broad energy range, for all transitions we studied. At intermediate and high energies the agreement is detailed and the results of two methods, basically, coincide. Not only is MQCT very accurate asymptotically, but also it predicts accurately the excitation thresholds. In the range of multiple scattering resonances, just above the threshold for each transition, it describes well the nonresonant background. We also carried out calculations for the "opposite" case, where the rotationally excited $\mathrm{N}_{2}(j=2)$ excites the ground state $\mathrm{H}_{2}(j=0)$, and we saw very similar behavior. ${ }^{63}$ We are also in the process of applying MQCT to transitions where the quantum states of both molecules change simultaneously, such as $(0,2) \rightarrow(2,0)$ transition. In this case the internal rotational energy of $\mathrm{H}_{2}(j=2)$ is used to excite $\mathrm{N}_{2}$ from $j=0$ to $j=2$. Another interesting example of this sort in the $\mathrm{N}_{2}+\mathrm{H}_{2}$ system is the $(20,2) \rightarrow(24,0)$ transition. In this special case energies of the initial and final rotational states of the entire system are nearly the same, with $\Delta E \approx 4.5 \mathrm{~cm}^{-1}$. So, during the collision process the internal energy of the system remains (nearly) the same, and the kinetic energy of the system does not change much. It is particularly interesting to test MQCT for such quasiresonant energy transfer processes, ${ }^{68,69}$ because they are known to be particularly efficient, for example, in such important systems as $\mathrm{H}_{2} \mathrm{O}+$ $\mathrm{H}_{2} \cdot{ }^{36}$ 


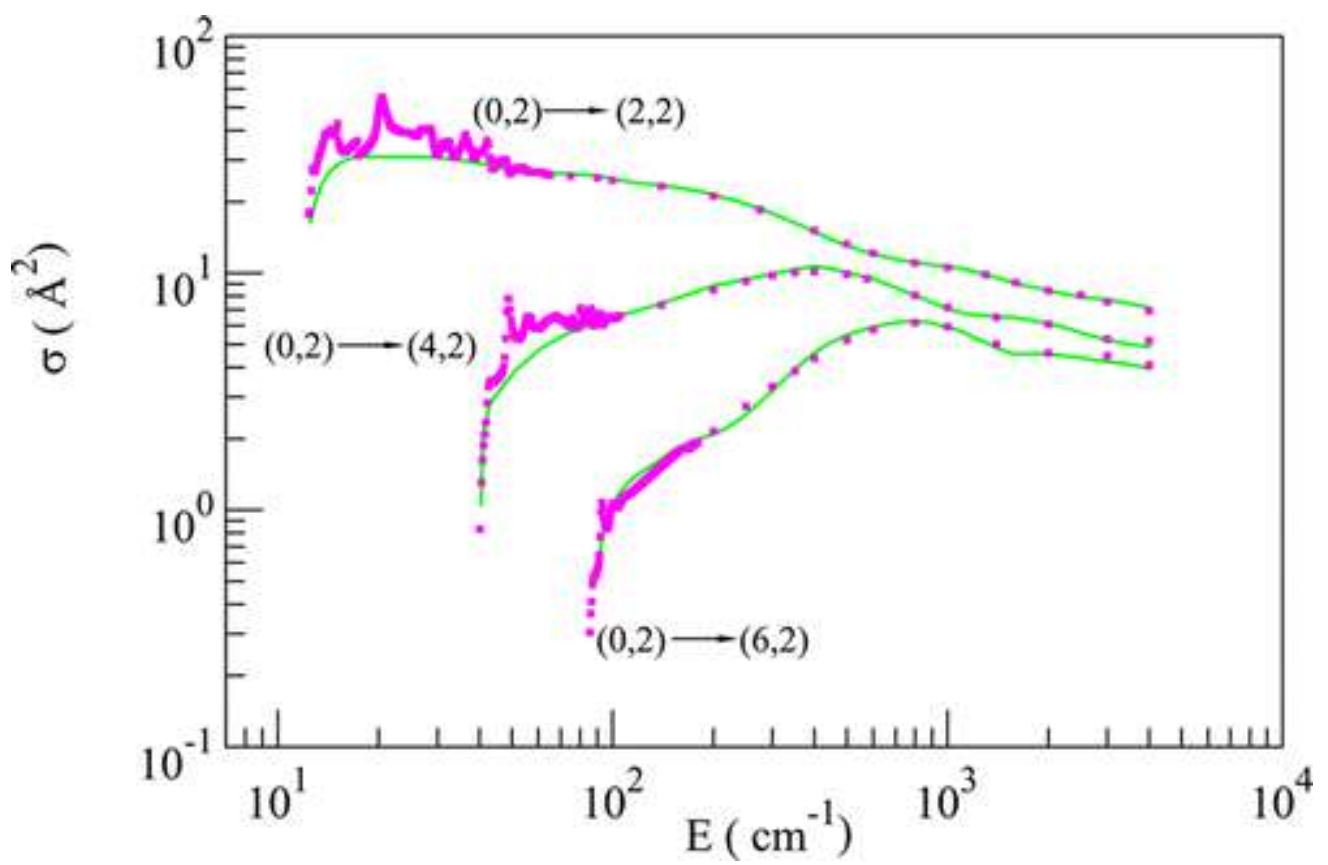

Figure 9. Excitation cross section for $\mathrm{N}_{2}+\mathrm{H}_{2}$. Full-quantum results are shown by symbols; the MQCT results are shown by lines. ${ }^{63}$ Initial and final states are labeled by $\left(j_{1} j_{2}\right)$ where first index belongs to $\mathrm{N}_{2}$ and the second index belongs to $\mathrm{H}_{2}$.

\section{4 Accuracy of MQCT}

One parameter that should be discussed for understanding the range of validity of MQCT is the de Broglie wavelength associated with masses and speeds of colliding partners. Qualitatively, we expect that MQCT is more accurate for more classical systems and collision conditions, described by shorter wavelengths that correspond to heavier masses and higher collision energies. For example, we saw that MQCT is more accurate in the cases of $\mathrm{N}_{2}+\mathrm{Na}$ and $\mathrm{HCOOCH}_{3}+$ $\mathrm{He}$ and is less accurate in the cases of $\mathrm{H}_{2}+\mathrm{He}$ and $\mathrm{CH}_{3}+\mathrm{He}$. To come out with some quantitative picture, we plotted the errors of MQCT (relative to the full-quantum results) versus a dimensionless ratio $R_{0} / \lambda$, where $R_{0}$ is the characteristic range of the molecule-quencher interaction potential (position of the minimum in the shallow van der Waals well in the asymptotic range), and $\lambda$ is the de Broglie wavelength (associated with the two-body reduced mass and the effective collision energy $U$ ). The data for different systems we studied so far were collected all together on the same plot. It should be mentioned, though, that the range of interaction $R_{0}$ does not change much from one system to another (here it varied from 6 Bohr in 
$\mathrm{HCOOCH}_{3}+\mathrm{He}$ to 10 Bohr in $\mathrm{N}_{2}+\mathrm{Na}$ ), whereas the de Broglie wavelength does change in a broad range, depending on collision energy and reduced mass. Figure 10 represents these data. We did not include those cases when other factors (such as scattering resonances, see below) seemed to have a dominant effect on the accuracy of MQCT. These data demonstrate some correlation between accuracy and the ratio $R_{0} / \lambda$. The less accurate corner in Figure 10, upper left, contains points that belong to lighter systems collided at lower energy. Here $R_{0} / \lambda \approx 5$ and the error can reach 30-40\%. Importantly, in the range of $R_{0} / \lambda>10$ the errors of MQCT drop exponentially (on average, over several different systems). The trend is such that near $R_{0} / \lambda \approx 20$ the error is on the order of 2 or $3 \%$.

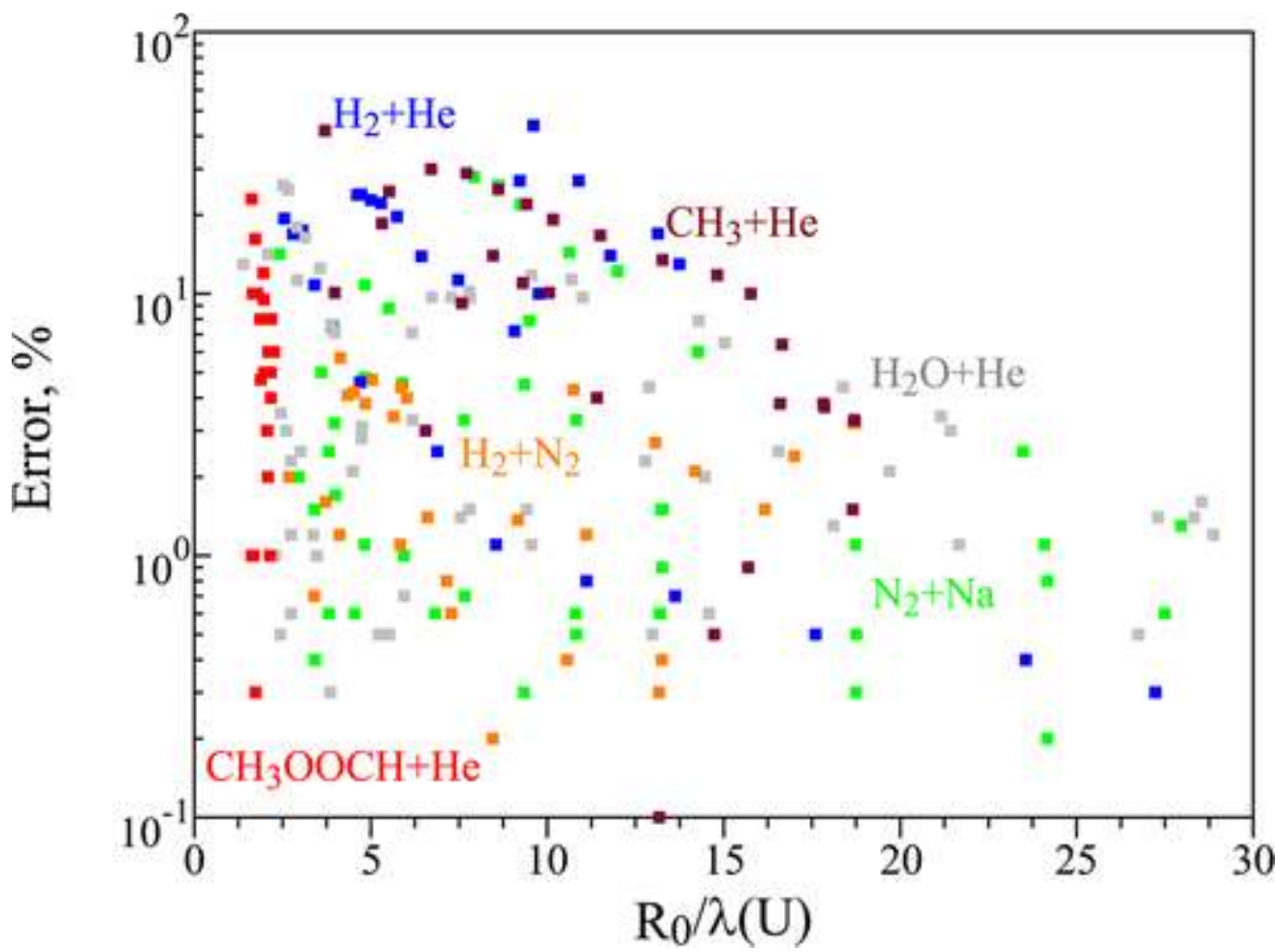

Figure 10. Errors of MQCT calculations (compared to the full-quantum results) as a function of the unitless ratio between the interaction range and the de Broglie wavelength. The data for six different systems are put together and are indicated by color.

Another consideration, sometimes referred to when the range of validity of MQCT is examined, is associated with Delos criterion. ${ }^{70}$ Formulated for electronic excitation in atom-atom collisions (not for molecular rotation), it states that a mixed quantum/classical 
description is generally valid when the energy change $\Delta E$ associated with quantum state-to-state transition is small compared to collision energy $E$. It appears that this criterion is too strict for rotational MQCT, because we often see good results even in the opposite limit, $E<\Delta E$, due to employment of the symmetrized (or average velocity) approach. The most striking example is quenching of state $j=22$ in $\mathrm{H}_{2}$ onto $j=20$, characterized by large $\Delta E=2968 \mathrm{~cm}^{-1}$ and accurately described by MQCT at collision energies of only $E \approx 350 \mathrm{~cm}^{-1} .60$ One reason for this anomalous accuracy is that this example describes quenching, not excitation. Indeed, we often see that MQCT results for quenching are rather good even at very low collision energies, because there is no threshold for the process, whereas results for excitation get worse near the threshold. One way to analyze MQCT results for both excitation and quenching on the same footing is to use the effective collision energy $U$ introduced above. Thus, our modified Delos criterion would include the ratio $\Delta E / U$. Figure 11 summarizes our data for different systems studied so far (except near resonances). Both excitation and quenching processes were included. The range of abscissa is rather broad and has the upper limit of $\triangle E / U=4$ achieved at $U=\Delta E / 4$, which corresponds to either excitation at the threshold energy $E=\Delta E$ or quenching with no energy, $E^{\prime}=0$ (section II.4 above). The points in Figure 11 spread quite a bit, especially in the more accurate (lower left) part of the graph, but still, this figure shows some correlation between accuracy of MQCT and the value of $\triangle E / U$, particularly in the less accurate (upper right) corner of the graph. Data for different molecules complement each other, following similar trends. The case of $\mathrm{H}_{2}+\mathrm{He}$ comes closest to the threshold $\Delta E / U=4$, where it demonstrates the worst accuracy, close to $40 \%$ (for transition from $j=22$ to $j=20$, characterized by large $\Delta E=2968 \mathrm{~cm}^{-1}$, at very low collision energies, near $2 \mathrm{~cm}^{-1}$ ). However, as the value of $\Delta E / U$ is reduced the error of MQCT drops exponentially and, on average, is on the order of only $3 \%$ in the range of $\triangle E / U \approx 1$, and on the order of $2 \%$ in the range of $\Delta E / U \approx 0.1$. Compared to the average (shown in Figure 11 by dotted line) the cases of $\mathrm{HCOOCH}_{3}+\mathrm{He}$ and $\mathrm{CH}_{3}+\mathrm{He}$ show somewhat larger errors. This is understood, because for the first of these two systems all the quantum-mechanical benchmark data ${ }^{12}$ belong to the very low-energy regime (where quantum resonances are still present), which complicates analysis. The second system is rather light and has large rotational quanta, similar to $\mathrm{H}_{2}+\mathrm{He}$. Overall, the 
dependencies of Figures 10 and 11 seem to be general and may be used to estimate the error of MQCT method before applying it to new systems.

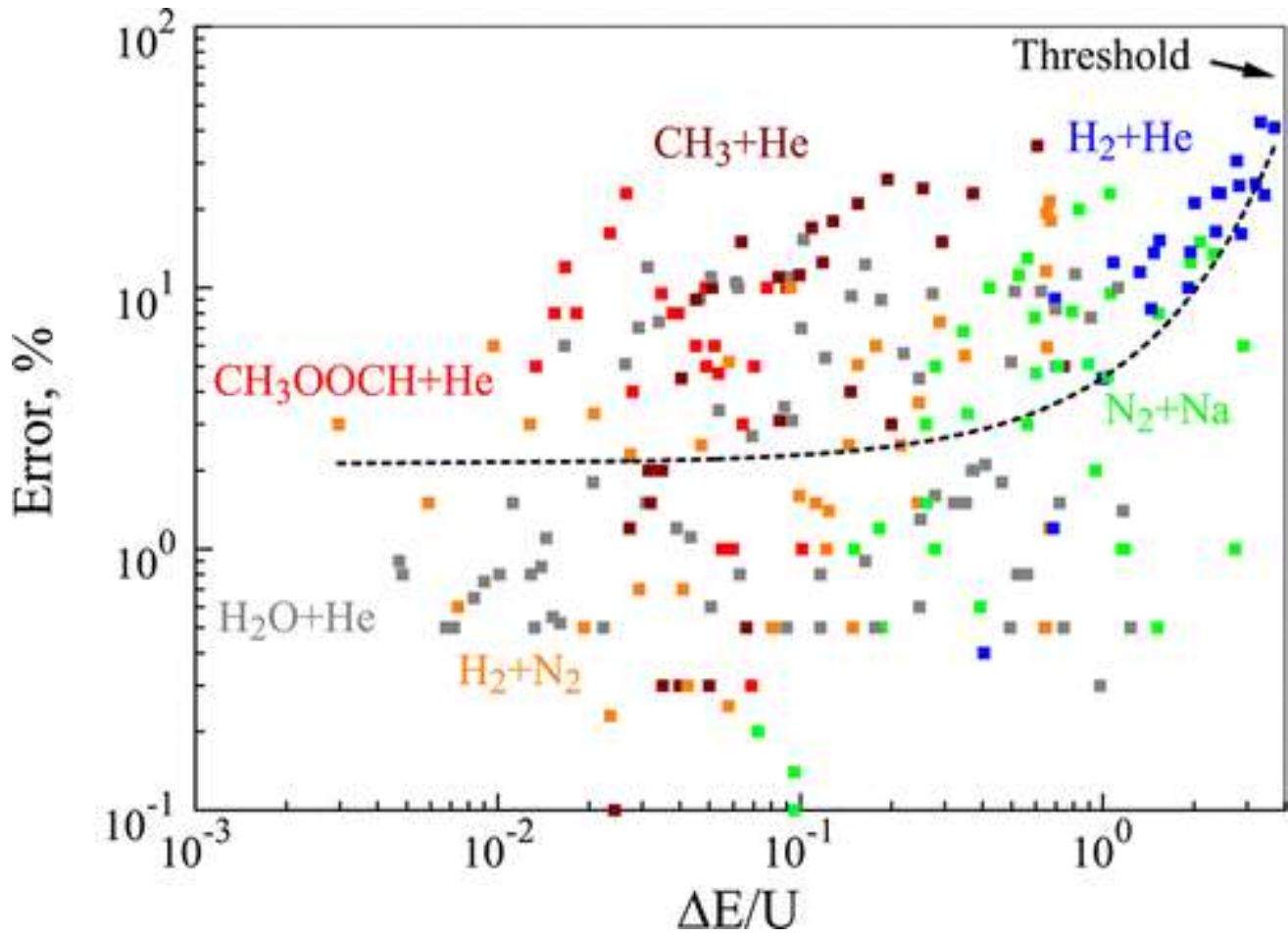

Figure 11. Errors of MQCT calculations (compared to the full-quantum results) as a function of the unitless ratio between the transition energy and the effective collision energy. The data for six different systems are put together and are indicated by color. The fit is by exponential function.

The next factor to consider is scattering resonances. We should admit that in the current implementation of MQCT we simply neglect the contribution of captured trajectories (orbiting), focusing on nonresonant or averaged behavior. In the cases where scattering resonances might be important or even dominant (e.g., lowtemperature reaction rates) the full-quantum description is, perhaps, indispensable. But the question whether MQCT is capable of treating scattering resonances is still open, and a solution may very well be found. In the early days of the quantum/classical theories this question has been addressed. ${ }^{41}$ We already showed that the quantum phase can be computed and used in MQCT (e.g., for calculations of differential cross sections), and we also plan to explore whether this phase information can help in description of scattering resonances. 
Finally, we saw that for one system, $\mathrm{CH}_{3}+\mathrm{He}$, some transitions are described less accurately than others (compare the upper and lower frames of Figure 6). We do not yet understand the reason for this effect. Because this is one system, the reduced masses and collision energies are the same for all these transitions. The values of $\Delta E$ and $U$ are also quite similar. So, neither $R_{0} / \lambda$ nor $\Delta E / U$ is expected to influence the process and be used to explain the difference. One possibility, which we are still checking, is the convergence issue (of either MQCT or the full-quantum results used as a reference), because it is known that some transitions may need larger basis sets and larger values of total $J_{\max }$ for convergence. More detailed information, such as comparison of MQCT vs full-quantum calculations at the level of opacity functions for each individual transition (e.g., at one chosen collision energy) may be needed to identify the reason for this disagreement. Detailed results and analysis for the $\mathrm{CH}_{3}+\mathrm{He}$ system will be reported elsewhere.

\section{5 Computational Cost of MQCT}

Quantum calculations for simple systems, such as diatomic + atom, are very fast, particularly using efficient codes such as MOLSCAT or Hibridon. Thus, computational advantages of MQCT can be seen clearly either when the collision energies are high or when the system is rather complicated. For example, quantum calculations ${ }^{12}$ for rotational excitation of methyl formate by He were affordable only at low collision energies, below $30 \mathrm{~cm}^{-1}$. But using MQCT, we ${ }^{62}$ were able to compute excitation cross sections at energies up to $1000 \mathrm{~cm}^{-1}$. (In principle, we could do MQCT calculations at even higher energies, but there we were limited by the accuracy of the PES representation, ${ }^{12}$ not really by the cost of MQCT calculations.)

It was also important to determine how the cost of MQCT calculations depends on the number of nondegenerate states (channels) included in the basis set expansion of eq 1 , the so-called scaling law. There are two ways of determining the scaling law. We could run calculations at fixed values of energy $E$ and $J_{\max }$, changing only the number of channels $n$ in a broad range (i.e., changing the basis set size only). Alternatively, we could determine the cost of calculations in a broad range of energies, converged at each energy 
with respect to the values of $J_{\max }$ and $n$. The first approach is better defined from the mathematical standpoint, but the second approach is more relevant physically, and represents better the actual cost of calculations. We tested MQCT in both ways for several molecules, ${ }^{61-63}$ and in all cases we found very similar scaling laws, on the order of $n^{2}$ or $n^{3}$. We also tested the full-quantum CC method using MOLSCAT for the case of $\mathrm{CH}_{3}+\mathrm{He}$. In the first (idealized) test we found the wellknown scaling law $n^{3}$, but in the second (more representative) test the scaling law was much worse, on the order of $n^{5}$ to $n^{6}$.

These results are interpreted in the following way: In the idealized test the scaling law of the full-quantum method is determined by the cost of matrix algebra, and the only variable is matrix size. However, in practice, when the energy is varied in a broad range (for example, is raised), the cost of the full-quantum calculations increases not only due to (i) increasing basis set size $n$ but also due to (ii) increasing number of partial scattering waves that have to be included for convergence, determined by the value of $J_{\max }$. The first contribution is described by the general law $n^{3}$, whereas the second contribution caries an overhead that is somewhat system-dependent but is anyway substantial, leading to the total cost approaching $n^{6}$. In contrast, MQCT has no such overhead, because scattering is treated classically. So, an increase of collision energy does not affect the scaling law of MQCT substantially, and it remains low, just $n^{2.5}$. This is the origin of high efficiency of MQCT at large scattering energies.

It must be recognized that one MQCT calculation from a given initial state produces only a column of the state-to-state matrix, not the entire scattering matrix. If the entire matrix is needed (which is rarely the case), the cost of MQCT will increase, by a factor of $n$ at most. However, calculations of different columns of the scattering matrix are entirely independent and could be done at the same time on different processors. Furthermore, reversibility can be employed to cut down the cost of these calculations, at least by a factor of 2 . The quantum parallelism can also be exploited, such as that in the timedependent wave packet calculations, by starting MQCT with the initial rotational wave packet, in contrast to the initial rotational eigenstate. 
Lastly, it should be stressed that MQCT methodology is intrinsically parallel. Different MQCT trajectories are entirely independent and can be propagated by different processors without any message passing. Only the final results from different trajectories are collected, to compute cross sections using eqs 9-11. Thus, the full power of massively parallel computers available today can be utilized.

\section{Conclusions}

We developed the mixed quantum/classical theory for inelastic scattering, which we named MQCT, in which only the relative scattering motion of two collision partners is treated classically, whereas the internal motion of each molecule is treated quantum mechanically. It can be applied to rotationally and vibrationally inelastic scattering and covers the molecule + atom and the molecule + molecule cases, including diatomic molecules, symmetric-top rotors, and the most general asymmetric-top rotor molecules. In this sense the theory is complete. Phase information is taken into consideration, which allows computing elastic and inelastic, total and differential cross sections. The scattering resonances (at very low collision energies) is one feature that requires further methodological development and testing of MQCT.

The method is numerically efficient and intrinsically parallel. Compared to the full-quantum calculations, the scaling law of MQCT is more favorable, which allows applying it to more complicated molecules and at higher collision energies. One representative example is rotational excitation of methyl formate by helium atoms, for which we computed cross sections at collision energies up to $1000 \mathrm{~cm}^{-1}$.

By comparing the results of MQCT against the full-quantum results for several quite different systems $\left(\mathrm{N}_{2}+\mathrm{Na}, \mathrm{H}_{2}+\mathrm{He}, \mathrm{CO}+\mathrm{He}\right.$, $\mathrm{CH}_{3}+\mathrm{He}, \mathrm{H}_{2} \mathrm{O}+\mathrm{He}, \mathrm{HCOOCH}_{3}+\mathrm{He}$, and $\mathrm{H}_{2}+\mathrm{N}_{2}$ ), we found that in many practically important scattering regimes the method is a viable approach to inelastic scattering. At higher collision energies it can confidently replace the full-quantum calculations that become computationally costly, if at all affordable. At low collision energies and for low-mass systems MQCT is less accurate, but even there it is not off by much. 
In fact, one could blend MQCT with full-quantum calculations in the following way: Typically, cross sections are needed in a broad range of collision energies. So, for low energies one should run the full-quantum calculations, because they are quite affordable in this regime and because scattering resonances may be present. These fullquantum results can be used as a benchmark for the subsequent MQCT calculations. If MQCT is found to be accurate enough (starting at some intermediate energy), then at higher energies one could continue calculations with MQCT only, because it is more affordable.

The authors declare no competing financial interest.

\section{Acknowledgment}

This research was partially supported by NSF, through the grant AGS1252486. Alexander Semenov acknowledges support of J. P. Raynor fellowship. We used resources of the National Energy Research Scientific Computing Center, which is supported by the Office of Science of the U.S. Department of Energy under Contract No. DE-AC02-5CH11231.

\section{References}

1. Roueff, E.; Lique, F.Molecular Excitation in the Interstellar Medium: Recent Advances in Collisional, Radiative, and Chemical Processes Chem. Rev. 2013, 113, 8906-8938, DOI: 10.1021/cr400145a

2. Yang, B.; Nagao, M.; Satomi, W.; Kimura, M.; Stancil, P. C.Rotational Quenching of Rotationally Excited $\mathrm{H} 2 \mathrm{O}$ in Collisions with He Astrophys. J. 2013, 765 (9) 77-77, DOI: 10.1088/0004-637X/765/2/77

3. Kirste, M.; Wang, X.; Schewe, H. C.; Meijer, G.; Liu, K.; van der Avoird, A. ; Janssen, L. M.; Gubbels, K. B.; Groenenboom, G. C.; van de Meerakker, S. Y.Quantum-State Resolved Bimolecular Collisions of Velocity-Controlled OH with NO Radicals Science 2012, 338, 10601063, DOI: $10.1126 /$ science. 1229549

4. Schewe, H. C.; Ma, Q.; Vanhaecke, N.; Wang, X.; Kłos, J.; Alexander, M. H.; van de Meerakker, S. Y. T.; Meijer, G.; van der Avoird, A.; Dagdigian, P. J.Rotationally Inelastic Scattering of $\mathrm{OH}$ by Molecular Hydrogen: Theory and Experiment J. Chem. Phys. 2015, 142 (13) 204310-204310, DOI: 10.1063/1.4921562

5. Dagdigian, P.; Alexander, M. H.Theoretical Investigation of Rotationally Inelastic Collisions of the Methyl Radical with Helium J. Chem. Phys. 2011, 135 (9) 064306-064306, DOI: 10.1063/1.3624525 
6. Tkáč, O.; Ma, Q.; Rusher, C. A.; Greaves, S. J.; Orr-Ewing, A. J.; Dagdigian, P. J.Differential and Integral Cross Sections for the Rotationally Inelastic Scattering Of Methyl Radicals With $\mathrm{H}_{2}$ And $\mathrm{D}_{2}$ J. Chem. Phys. 2014, 140 (12) 204318-204318, DOI: 10.1063/1.4879618

7. Balakrishnan, N.; Groenenboom, G. C.; Krems, R. V.; Dalgarno, A.The He$\mathrm{CaH}\left({ }^{2} \Sigma^{+}\right)$Interaction. II. Collisions at Cold and Ultracold Temperatures J. Chem. Phys. 2003, 118, 7386-7393, DOI: 10.1063/1.1562947

8. Mack, A.; Clark, T. K.; Forrey, R. C.; Balakrishnan, N.; Lee, T.-G.; Stancil, P. C.Cold $\mathrm{He}+\mathrm{H}_{2}$ Collisions near Dissociation Phys. Rev. A: At., Mol., Opt. Phys. 2006, 74 (8) 052718, DOI: 10.1103/PhysRevA.74.052718

9. Wernli, M.; Wiesenfeld, L.; Faure, A.; Valiron, P.Rotational excitation of $\mathrm{HC}_{3} \mathrm{~N}$ by $\mathrm{H}_{2}$ and $\mathrm{He}$ at low temperatures Astron. Astrophys. 2007, 464, 1147-1154, DOI: 10.1051/0004-6361:20066112

10. Troscompt, N.; Faure, A.; Wiesenfeld, L.; Ceccarelli, C.; Valiron, P.Rotational Excitation of Formaldehyde by Hydrogen Molecules: Ortho- $\mathrm{H}_{2} \mathrm{CO}$ at Low Temperature Astron. Astrophys. 2009, 493, 687696, DOI: $10.1051 / 0004-6361: 200810712$

11. Rabli, D.; Flower, D. R.The Rotational Excitation of Methanol by Molecular Hydrogen Mon. Not. R. Astron. Soc. 2010, 406, 95, DOI:

10.1111/j.1365-2966.2010.16671.x

12. Faure, A.; Szalewicz, K.; Wiesenfeld, L.Potential Energy Surface and Rotational Cross Sections for Methyl Formate Colliding with Helium J. Chem. Phys. 2011, 135 (10) 024301-024301, DOI: 10.1063/1.3607966

13. Braams, B.; Bowman, J.Permutationally Invariant Potential Energy Surfaces in High Dimensionality Int. Rev. Phys. Chem. 2009, 28, 577-606, DOI: 10.1080/01442350903234923

14. Xie, Z.; Bowman, J.Permutationally Invariant Polynomial Basis for Molecular Energy Surface Fitting via Monomial Symmetrization J. Chem. Theory Comput. 2010, 6, 26-34, DOI: 10.1021/ct9004917

15. Ma, Q.; van der Avoird, A.; Loreau, J.; Alexander, M. H.; van de Meerakker, S. Y. T.; Dagdigian, P. J.Resonances in Rotationally Inelastic Scattering of $\mathrm{NH}_{3}$ and $\mathrm{ND}_{3}$ with $\mathrm{H}_{2}$ J. Chem. Phys. 2015, 143 (10) 044312, DOI: $10.1063 / 1.4927074$

16. Faure, A.; Josselin, E.Collisional Excitation of Water in Warm Astrophysical Media: I. Rate Coefficients for Ro-Vibrationally Excited States Astron. Astrophys. 2008, 492, 257-264, DOI: 10.1051/0004-6361:200810717

17. dos Santos, F. S.; Balakrishnan, N.; Forrey, R. C.; Stancil, P. C.Vibrationvibration and Vibration-translation Energy Transfer in $\mathrm{H}_{2}-\mathrm{H}_{2}$ Collisions: a Critical Test of Experiment with Full-Dimensional Quantum Dynamics J. Chem. Phys. 2013, 138 (10) 104302-104302, DOI: $10.1063 / 1.4793472$ 
18. Song, H.; Lu, Y.; Lee, S.-Y.Fully Converged Integral Cross Sections of Collision Induced Dissociation, Four-Center, and Single Exchange Reactions, and Accuracy of the Centrifugal Sudden Approximation, in $\mathrm{H}_{2}+\mathrm{D}_{2}$ Reaction J. Chem. Phys. 2012, 136 (9) 114307-114307, DOI: $10.1063 / 1.3693762$

19. Yang, C. H.; Sarma, G.; Parker, D. H.; ter Meulen, J. J.; Wiesenfeld, L.State-to-state Differential and Relative Integral Cross Sections for Rotationally Inelastic Scattering of $\mathrm{H}_{2} \mathrm{O}$ by Hydrogen J. Chem. Phys. 2011, 134 (12) 204308-204308, DOI: 10.1063/1.3589360

20. Loreau, J.; Zhang, P.; Dalgarno, A.Elastic Scattering and Rotational Excitation of Nitrogen Molecules by Sodium Atoms J. Chem. Phys. 2011, 135 (8) 174301-174301, DOI: 10.1063/1.3653983

21. Barker, J. R.; Yoder, L. M.; King, K. D.Vibrational Energy Transfer Modeling of Nonequilibrium Polyatomic Reaction Systems J. Phys. Chem. A 2001, 105, 796-809, DOI: 10.1021/jp002077f

22. Barker, J. R.; Weston, R. E.Collisional Energy Transfer Probability Densities $\mathrm{P}\left(\mathrm{E}, \mathrm{J} ; \mathrm{E}^{\prime}, \mathrm{J}^{\prime}\right)$ For Monatomics Colliding with Large Molecules J. Phys. Chem. A 2010, 114, 10619-10633, DOI: 10.1021/jp106443d

23. Houston, P. L.; Conte, R.; Bowman, J. M.Collisional Energy Transfer in Highly Excited Molecules J. Phys. Chem. A 2014, 118, 7758, DOI: $10.1021 / j p 506202 \mathrm{~g}$

24. Lehman, J. H.; Dempsey, L. P.; Lester, M. I.; Fu, B.; Kamarchik, E.; Bowman, J. M.Collisional Quenching of OD A ${ }^{2} \Sigma^{+}$by $\mathrm{H}_{2}$ : Experimental and Theoretical Studies Of The State-Resolved OD X ${ }^{2} \Pi$ Product Distribution and Branching Fraction J. Chem. Phys. 2010, 133 (9) 164307-164307, DOI: 10.1063/1.3487734

25. Conte, R.; Houston, P. L.; Bowman, J. M.Trajectory Study of Energy Transfer and Unimolecular Dissociation of Highly Excited Allyl with Argon J. Phys. Chem. A 2014, 118, 7742-7757, DOI: 10.1021/jp5062013

26. Jasper, A. W.; Miller, J. A.Theoretical Unimolecular Kinetics for $\mathrm{CH}_{4}+\mathrm{M} \leftrightarrow$ $\mathrm{CH}_{3}+\mathrm{H}+\mathrm{M}$ in Eight Baths, $\mathrm{M}=\mathrm{He}, \mathrm{Ne}, \mathrm{Ar}, \mathrm{Ke}, \mathrm{H}_{2}, \mathrm{~N}_{2}, \mathrm{CO}$ And $\mathrm{CH}_{4} \mathrm{~J}$. Phys. Chem. A 2011, 115, 6438-6455, DOI: 10.1021/jp200048n

27. Czako, G.; Bowman, J.Reaction Dynamics of Methane with $\mathrm{F}, \mathrm{O}, \mathrm{Cl}$, and $\mathrm{Br}$ On Ab Initio Potential Energy Surfaces J. Phys. Chem. A 2014, 118, 2839-2864, DOI: 10.1021/jp500085h

28. Brunsvold, A. L.; Garton, D. J.; Minton, T. K.; Troya, D.; Schatz, G. C.Crossed Beams and Theoretical Studies of the Dynamics of Hyperthermal Collisions between Ar and Ethane J. Chem. Phys. 2004, 121, 11702-11714, DOI: $10.1063 / 1.1815271$

29. Schinke, R.; Fleurat-Lessard, P.The Effect of Zero-Point Energy Differences on the Isotope Dependence of The Formation of Ozone: aClassical 
Trajectory Study J. Chem. Phys. 2005, 122 (9) 094317-094317, DOI: $10.1063 / 1.1860011$

30. Czakó, G.; Kaledin, A. L.; Bowman, J. M.A Practical Method to Avoid ZeroPoint Leak in Molecular Dynamics Calculations: Application to the Water Dimer J. Chem. Phys. 2010, 132 (6) 164103-164103, DOI: $10.1063 / 1.3417999$

31. Varandas, A. J. C.Trajectory Binning Scheme And Non-Active Treatment of Zero-Point Energy Leakage in Quasi-Classical Dynamics Chem. Phys. Lett. 2007, 439, 386-392, DOI: 10.1016/j.cplett.2007.03.090

32. Babikov, D.; Walker, R. B.; Pack, R. T.A Quantum Symmetry Preserving Semiclassical Method J. Chem. Phys. 2002, 117, 8613-8622, DOI: 10.1063/1.1513457

33. Wang, Y.; Bowman, J. M.Mode-specific Tunneling Using the $Q_{\mathrm{im}}$ Path: Theory and an Application to Full-Dimensional Malonaldehyde J. Chem. Phys. 2013, 139 (5) 154303- 154303, DOI: 10.1063/1.4824713

34. Arthurs, A. M.; Dalgarno, A.The Theory of Scattering by a Rigid Rotator Proc. R. Soc. London, Ser. A 1960, 256, 540-551, DOI: 10.1098/rspa.1960.0125

35. Green, S.Rotational Excitation of Symmetric Top Molecules by Collisions with Atoms: Close Coupling, Coupled States, and Effective Potential Calculations for $\mathrm{NH}_{3}-\mathrm{He}$ J. Chem. Phys. 1976, 64, 3463-3473, DOI: $10.1063 / 1.432640$

36. Daniel, F.; Dubernet, M.-L.; Grosjean, A.Rotational excitation of 45 levels of ortho/para- $\mathrm{H}_{2} \mathrm{O}$ by excited ortho/para- $\mathrm{H}_{2}$ from 5 to $1500 \mathrm{~K}$ : stateto-state, effective, and thermalized rate coefficients Astron. Astrophys. 2011, A76, 536-546, DOI: 10.1051/0004-6361/201118049

37. Faure, A.; Wiesenfeld, L.; Scribano, Y.; Ceccarelli, C.Rotational Excitation of Mono- and Doubly-Deuterated Water by Hydrogen Molecules Mon. Not. R. Astron. Soc. 2012, 420, 699- 704, DOI: 10.1111/j.13652966.2011.20081.x

38. Agg, P. J.; Clary, D. C.Infinite Order Sudden Calculation of Raman QBranch Linewidths for $\mathrm{H}_{2} \mathrm{O}+\mathrm{H}_{2} \mathrm{O}$ J. Chem. Phys. 1991, 95, 10371048, DOI: $10.1063 / 1.461131$

39. McCann, K. J.; Flannery, M. R.A Multistate Semiclassical Orbital Treatment of Heavy-Particle Collisions with Application to $\mathrm{H}+\mathrm{H}_{2}$ Rotational Transitions Chem. Phys. Lett. 1975, 35, 124-130, DOI: 10.1016/0009-2614(75)85601-6

40. McCann, K. J.; Flannery, M. R.New Semiclassical Treatments of Rotational and Vibrational Transitions in Heavy-Particle Collisions. I. $\mathrm{H}-\mathrm{H}_{2}$ and $\mathrm{He}-\mathrm{H}_{2}$ Collisions J. Chem. Phys. 1975, 63, 4695-4707, DOI: $10.1063 / 1.431255$ Society and permission has been granted for this version to appear in e-Publications@Marquette. American Chemical Society does not grant permission for this article to be further copied/distributed or hosted elsewhere without the express permission from American Chemical Society. 
NOT THE PUBLISHED VERSION; this is the author's final, peer-reviewed manuscript. The published version may be

accessed by following the link in the citation at the bottom of the page.

41. McCann, K. J.; Flannery, M. R.Elastic Scattering and Rotational Excitation in Ion-Molecule Collisions. II. $\mathrm{Li}^{+}-\mathrm{H}_{2}$ and $\mathrm{H}^{+}-\mathrm{H}_{2}$ Collisions J. Chem. Phys. 1978, 69, 5275-5287, DOI: 10.1063/1.436581

42. Billing, G. D.The Semiclassical Treatment of Molecular Roto-Vibrational Energy Transfer Comput. Phys. Rep. 1984, 1, 239-296, DOI: 10.1016/0167-7977(84)90006-6

43. Billing, G. D. The Quantum-Classical Theory; Oxford University Press: Oxford, U.K., 2002.

44. Babikov, D.; Aguillon, F.; Sizun, M.; Sidis, V.Fragmentation Of $\mathrm{Na}_{2}{ }^{+}$Dimer Ions in Kilo-electron-volt Collisions with He: A Coupled Wave-packet Study Phys. Rev. A: At., Mol., Opt. Phys. 1999, 59, 330-341, DOI: 10.1103/PhysRevA.59.330

45. Whittier, G. S.; Light, J. C.Quantum/Classical Time-Dependent SelfConsistent Field Treatment of $\mathrm{Ar}+\mathrm{HCO}$ Inelastic and Dissociative Scattering J. Chem. Phys. 1999, 110, 4280-4290, DOI: $10.1063 / 1.478291$

46. Ermoshin, V. A.; Kazansky, A. K.; Engel, V.A Novel Mixed Quantum/Classical Method for the Dynamics of Molecules in a Gas Environment with an Application to Femtosecond Time-Resolved Spectroscopy Chem. Phys. Lett. 1999, 302, 20-26, DOI: 10.1016/S0009-2614(99)00086-X

47. Tully, C.; Johnson, R. E.Semiclassical Calculation of Collisional Dissociation Cross Sections for $\mathrm{N}+\mathrm{N}_{2}$ J. Chem. Phys. 2002, 117, 6556-6561, DOI: 10.1063/1.1504085

48. Chen, F.; McCoy, A. B.Multiple-Configuration Quantum/Classical Studies of the Photodissociation Dynamics of $\mathrm{H}_{2} \mathrm{O}$ J. Phys. Chem. A 2003, 107, 7220-7229, DOI: 10.1021/jp034498d

49. Thompson, W. H.A General Method for Implementing Vibrationally Adiabatic Mixed Quantum-Classical Simulations J. Chem. Phys. 2003, 118, 1059-1067, DOI: 10.1063/1.1528891

50. Avery, J.; Herman, Z.Review Article: Gert Due Billing (1946-2002) Chem. Soc. Rev. 2003, 32, iv, DOI: 10.1039/B212398N

51. Ivanov, M. V.; Babikov, D.Mixed Quantum-Classical Theory for The Collisional Energy Transfer and The Ro-Vibrational Energy Flow: Application to Ozone Stabilization J. Chem. Phys. 2011, 134 (16) 144107-144107, DOI: $10.1063 / 1.3576103$

52. Ivanov, M. V.; Babikov, D.Efficient Quantum-Classical Method for Computing Thermal Rate Constant of Recombination: Application to Ozone Formation J. Chem. Phys. 2012, 136 (16) 184304-184304, DOI: $10.1063 / 1.4711760$

53. Ivanov, M. V.; Babikov, D.On Molecular Origin of Mass-Independent Fractionation of Oxygen Isotopes in the Ozone Forming Recombination

Journal of Physical Chemistry A, Vol 120, No. 3 (January 28, 2016): pg. 319-331. DOI. This article is @ American Chemical Society and permission has been granted for this version to appear in e-Publications@Marquette. American Chemical Society does not grant permission for this article to be further copied/distributed or hosted elsewhere without the express permission from American Chemical Society. 
NOT THE PUBLISHED VERSION; this is the author's final, peer-reviewed manuscript. The published version may be

accessed by following the link in the citation at the bottom of the page.

Reaction Proc. Natl. Acad. Sci. U. S. A. 2013, 110 (5) 17708-17708, DOI: $10.1073 /$ pnas.1215464110

54. Semenov, A.; Babikov, D.Equivalence of The Ehrenfest Theorem snd The Fluid-Rotor Model for Mixed Quantum/Classical Theory of Collisional Energy Transfer J. Chem. Phys. 2013, 138 (10) 164110-164110, DOI: $10.1063 / 1.4801430$

55. Semenov, A.; Ivanov, M. V.; Babikov, D.Ro-vibrational Quenching of CO ( $=1$ ) by He Impact in a Broad Range of Temperatures: A Benchmark Study Using Mixed Quantum/Classical Inelastic Scattering Theory J. Chem. Phys. 2013, 139 (12) 074306-074306, DOI:

$10.1063 / 1.4818488$

56. Billing, G. D.Comparison of Quantum Mechanical and Semiclassical Cross Sections for Rotational Excitation of Hydrogen Chem. Phys. Lett. 1977, 50, 320-323, DOI: 10.1016/0009-2614(77)80190-5

57. Semenov, A.; Babikov, D.Mixed Quantum/Classical Theory of Rotationally and Vibrationally Inelastic Scattering in Space-fixed And Body-fixed Reference Frames J. Chem. Phys. 2013, 139 (15) 174108-174108, DOI: $10.1063 / 1.4827256$

58. Ivanov, M. V.; Dubernet, M.-L.; Babikov, D. Rotational Quenching of $\mathrm{H}_{2} \mathrm{O}$ by He: Mixed Quantum/Classical Theory and Comparison with Quantum Results J. Chem. Phys. 2014, 140 (7) 134301-134301, DOI: $10.1063 / 1.4868715$

59. Semenov, A.; Babikov, D.Accurate Calculations of Rotationally Inelastic Scattering Cross Sections Using Mixed Quantum/Classical Theory J. Phys. Chem. Lett. 2014, 5, 275-278, DOI: 10.1021/jz402542w

60. Semenov, A.; Babikov, D.Mixed Quantum/Classical Calculations of Total and Differential Elastic and Rotationally Inelastic Scattering Cross Sections for Light and Heavy Reduced Masses in a Broad Range of Collision Energies J. Chem. Phys. 2014, 140 (13) 044306, DOI: 10.1063/1.4862409

61. Semenov, A.; Dubernet, M.-L.; Babikov, D.Mixed Quantum/Classical Theory for Inelastic Scattering of Asymmetric-Top-Rotor + Atom in The Body-fixed Reference Frame and Application to the $\mathrm{H}_{2} \mathrm{O}+\mathrm{He}$ System J. Chem. Phys. 2014, 141 (8) 114304, DOI: 10.1063/1.4895607

62. Semenov, A.; Babikov, D.Mixed Quantum/Classical Approach for Description of Molecular Collisions in Astrophysical Environments J. Phys. Chem. Lett. 2015, 6, 1854-1858, DOI: 10.1021/acs.jpclett.5b00496

63. Semenov, A.; Babikov, D.Mixed Quantum/Classical Theory for MoleculeMolecule Inelastic Scattering: Derivations of Equations and Application to $\mathrm{N}_{2}+\mathrm{H}_{2}$ System J. Phys. Chem. A 2015, , DOI: 10.1021/acs.jpca.5b06812

Journal of Physical Chemistry A, Vol 120, No. 3 (January 28, 2016): pg. 319-331. DOI. This article is @ American Chemical Society and permission has been granted for this version to appear in e-Publications@Marquette. American Chemical Society does not grant permission for this article to be further copied/distributed or hosted elsewhere without the express permission from American Chemical Society. 
NOT THE PUBLISHED VERSION; this is the author's final, peer-reviewed manuscript. The published version may be accessed by following the link in the citation at the bottom of the page.

64. Hutson, J. M.; Green, S. MOLSCAT Computer Code, version 14; distributed by Collaborative Computational Project No. 6 of the Engineering and Physical Sciences Research Council, Swindon, U.K., 1994.

65. Alexander, M. H.; Manolopoulos, D. E.; Werner, H.-J.; Follmeg, B. HIBRIDON, available at http://www2.chem.umd.edu/groups/alexander/hibridon/hib43/hibhelp. html (last accessed on 11/3/2015).

66. Levine, R. D. Quantum Mechanics of Molecular Rate Processes; Oxford University Press: Oxford, U.K., 1969.

67. Gomez, L.; Bussery-Honvault, B.; Cauchy, T.; Bartolomei, M.; Cappelletti, D.; Pirani, F.Global Fits of New Intermolecular Ground State Potential Energy Surfaces for $\mathrm{N}_{2}-\mathrm{H}_{2}$ and $\mathrm{N}_{2}-\mathrm{N}_{2}$ Van Der Waals Dimers Chem. Phys. Lett. 2007, 445, 99-107, DOI: 10.1016/j.cplett.2007.07.053

68. Lanza, M.; Kalugina, Y.; Wiesenfeld, L.; Lique, F.Near-Resonant Rotational Energy Transfer in $\mathrm{HCl}-\mathrm{H}_{2}$ Inelastic Collisions J. Chem. Phys. 2014, 140, 064316-064316-10, DOI: 10.1063/1.4864359

69. Yang, B.; Zhang, P.; Wang, X.; Stancil, P. C.; Bowman, J. M.; Balakrishnan, N.; Forrey, R. C.Quantum Dynamics of $\mathrm{CO}-\mathrm{H}_{2}$ in Full Dimensionality Nat. Commun. 2015, 6 (1-10) 6629, DOI: $10.1038 /$ ncomms7629

70. Delos, J. B.; Thorson, W. R.; Knudson, S. K.Semiclassical Theory of Inelastic Collisions. I. Classical Picture and Semiclassical Formulation Phys. Rev. A: At., Mol., Opt. Phys. 1972, 6, 709-720, DOI: 10.1103/PhysRevA.6.709

Journal of Physical Chemistry A, Vol 120, No. 3 (January 28, 2016): pg. 319-331. DOI. This article is @ American Chemical Society and permission has been granted for this version to appear in e-Publications@Marquette. American Chemical Society does not grant permission for this article to be further copied/distributed or hosted elsewhere without the express permission from American Chemical Society. 